\title{
The Effect of Dietary Supplements on Endurance Exercise Performance and Core Temperature in Hot Environments: A Meta-analysis and Meta-regression
}

\author{
Jennifer S. Peel ${ }^{1}$ (D) - Melitta A. McNarry ${ }^{1} \cdot$ Shane M. Heffernan $^{1} \cdot$ Venturino R. Nevola $^{1,2} \cdot$ Liam P. Kilduff $^{1,3}$. \\ Mark Waldron ${ }^{1,3,4}$
}

Accepted: 2 June 2021 / Published online: 15 June 2021

(C) The Author(s) 2021

\begin{abstract}
Background The ergogenic effects of dietary supplements on endurance exercise performance are well-established; however, their efficacy in hot environmental conditions has not been systematically evaluated.

Objectives (1) To meta-analyse studies investigating the effects of selected dietary supplements on endurance performance and core temperature responses in the heat. Supplements were included if they were deemed to: (a) have a strong evidence base for 'directly' improving thermoneutral endurance performance, based on current position statements, or (b) have a proposed mechanism of action that related to modifiable factors associated with thermal balance. (2) To conduct metaregressions to evaluate the moderating effect of selected variables on endurance performance and core temperature responses in the heat following dietary supplementation.

Methods A search was performed using various databases in May 2020. After screening, 25 peer-reviewed articles were identified for inclusion, across three separate meta-analyses: (1) exercise performance; (2) end core temperature; (3) submaximal core temperature. The moderating effect of several variables were assessed via sub-analysis and meta-regression. Results Overall, dietary supplementation had a trivial significant positive effect on exercise performance (Hedges' $g=0.18$, 95\% CI 0.007-0.352, $P=0.042$ ), a trivial non-significant positive effect on submaximal core temperature (Hedges' $g=0.18$, $95 \% \mathrm{CI}-0.021$ to $0.379, P=0.080$ ) and a small non-significant positive effect on end core temperature (Hedges' $g=0.20$, $95 \%$ CI -0.041 to $0.439, P=0.104)$ in the heat. There was a non-significant effect of individual supplements on exercise performance $(P=0.973)$ and submaximal core temperature $(P=0.599)$. However, end core temperature was significantly affected by supplement type ( $P=0.003)$, which was attributable to caffeine's large significant positive effect ( $n=8$; Hedges' $g=0.82,95 \%$ CI $0.433-1.202, P<0.001)$ and taurine's medium significant negative effect $(n=1$; Hedges' $g=-0.96,95 \%$ CI -1.855 to $-0.069, P=0.035$ ).

Conclusion Supplements such as caffeine and nitrates do not enhance endurance performance in the heat, with caffeine also increasing core temperature responses. Some amino acids might offer the greatest performance benefits in the heat. Exercising in the heat negatively affected the efficacy of many dietary supplements, indicating that further research is needed and current guidelines for performance in hot environments likely require revision.
\end{abstract}

Jennifer S. Peel

856558@swansea.ac.uk

1 A-STEM Centre, College of Engineering, Swansea University, Swansea, UK

2 Defence Science and Technology Laboratory (Dstl), Fareham, Hampshire, UK

3 Welsh Institute of Performance Science, Swansea University, Swansea, UK

4 School of Science and Technology, University of New England, Armidale, NSW, Australia 


\section{Key Points}

Exercising in the heat appears to affect the efficacy of many dietary supplements, suggesting that findings from research conducted on certain supplements in thermoneutral conditions are not necessarily transferrable to other environmental conditions.

Certain supplements, such as caffeine and nitrate, lack sufficient data to support their use as ergogenic aids in the heat, despite their efficacy in thermoneutral conditions, with caffeine also increasing core temperature responses. Some amino acids might offer the greatest performance benefits in the heat.

A potential risk is posed to those in physical performance domains (i.e., athletes or military personnel) due to the limited guidance on how to supplement appropriately for endurance exercise in hot environments.

\section{Introduction}

The ergogenic effects of a number of dietary supplements on endurance exercise performance are well-established [1-5]. Indeed, recent position statements by the International Olympic Committee (IOC [6]), American College of Sports Medicine (ACSM [7]) and the Union of European Football Associations (UEFA [8]) provide specific recommendations for certain performance enhancing dietary supplements that are thought to have sufficient evidence for use by endurance athletes during training and competition. In tactical occupational settings, official legal information on the use of dietary supplements is often provided [9]; however, specific guidance on ergogenic aids is not. Despite this, the use of supplements among military personnel in training $[10,11]$ and during operations $[12,13]$ has been well reported. While it has been recognised that contextual factors should be considered when selecting dietary supplements [6,9], there is limited guidance on this relating to endurance exercise performed in hot environments. This is particularly surprising, given that many endurance events and major international competitions take place in a combination of hot and humid conditions $[14,15]$. For example, the forthcoming Tokyo 2021 Olympic Games are expected to take place in air temperatures exceeding $30{ }^{\circ} \mathrm{C}$, with a humidity index of $\sim 38$ $[16,17]$. Furthermore, military training and operations are also often conducted in extreme environments, in combination with prolonged endurance activity $[18,19]$.
Physical capacity is markedly impaired with increasing ambient temperature and humidity [20-23], leading to thermoregulatory strain and early onset fatigue, for a variety of physiological reasons [20, 24-32]. To perform optimally, environmental conditions - and their interaction with dietary supplement choices-must be carefully considered. Improper preparation for exercise in the heat can not only have detrimental effects on performance but can also lead to severe heat illness, and even death, in some extreme cases [33-36]. Therefore, a more comprehensive understanding of the effects of commonly used dietary supplements on physical performance and thermoregulation during exercise in the heat is necessary and could lead to safer and/or more efficacious heat preparation strategies.

The major limiting factors during exercise in the heat are linked to inexorable increases in core temperature [25], cardiovascular strain [29] and/or reductions in central drive [30]. Conceptually, regarding most endurance athletes and military personnel, the capacity to dissipate heat and offset one, or all, of these eventualities in hot environments predominantly occurs via three modifiable factors: lowered metabolic heat production, enhanced skin vasodilation (i.e., convective heat loss) or evaporative heat transfer (i.e., sweating [37-39]). The two supplements deemed to have the strongest empirical evidence to support these mechanisms [6], and reportedly serve to aid endurance exercise performance in temperate conditions, are caffeine (1,3,7-trimethylxanthine $[1,40])$ and dietary nitrate $\left(\mathrm{NO}_{3}{ }^{-}[4]\right)$. Mechanistically, there is a sound theoretical basis for both caffeine and $\mathrm{NO}_{3}{ }^{-}$supplementation to offset fatigue in the heat through increased central drive (caffeine [41]), and nitric oxide's (NO) action on eccrine sweat gland function and subcutaneous microvascular control $\left(\mathrm{NO}_{3}{ }^{-}[42-44]\right)$. However, numerous studies have reported negative or null performance and thermoregulatory effects for both of these supplements during exercise in the heat [45-49].

The apparent failure of these well-evidenced supplements to produce an ergogenic effect in the heat is largely unexplained but could be due to the differing physiological demands of exercise in the heat, and a combination of factors limiting exercise tolerance in a hot environment [26-31]. It is also possible that ancillary physiological effects (i.e., on core temperature and blood pressure) of selected supplements have not been fully considered in accordance with environmental constraints and could inadvertently exacerbate symptoms of heat stress, which has been inferred from laboratory-based studies of caffeine [48] and $\mathrm{NO}_{3}{ }^{-}$supplementation [50]. A similar line of reasoning can be applied to most other dietary supplements, based on the poor knowledge of their specific effects on thermoregulatory processes and subsequent ergogenic effects in the heat. Indeed, a number of alternative supplements have received some attention for their use in hot environments. For instance, 
supplementation with branched-chain amino acids (BCAAs [51]), tyrosine [52] and taurine [53] has been shown to extend time-to-exhaustion (TTE) in the heat, indicating that amino acids (AA) have ergogenic potential in hot conditions, yet these are not among those most commonly selected for training or competition purposes $[54,55]$. Irrespective of the exact reasons for the apparent inconsistent findings within the published literature, there has not yet been a systematic evaluation of dietary supplements for endurance athletes and/or military personnel in the heat, which is necessary to clarify the most ergogenic options and those least likely to contribute to rises in core temperature.

Therefore, the aims of the current meta-analysis were to investigate the effects of selected dietary supplements on endurance performance in the heat, as well as the associated core temperature responses. The ergogenic effect of macronutrients [56-58] and eu/hyper-hydration [56, 59-64] on endurance exercise performance in the heat have been well-established and do not require revisiting here. However, the control of these factors among studies evaluating the efficacy of dietary supplements can be inconsistent, often precluding direct comparisons. Likewise, the training and acclimation status of participants has a significant effect on their thermoregulatory control and subsequent heat tolerance [65], as does the selected mode of exercise (i.e., time trial (TT) vs TTE [66]). This will affect behaviour and pacing during performance [67], yet these details appear to lack appropriate attention and have been largely overlooked in current consensus guidelines [6, 7]. Therefore, to understand the potential effects of dietary supplements on endurance performance in the heat, these factors were considered as potential moderating variables, forming part of the current meta-regression analysis.

\section{Methods}

\subsection{Search Strategy}

All literature that investigated the effects of dietary supplementation on exercise performance in a hot environment was searched and obtained using the Preferred Reporting Items for Systematic Reviews and Meta-analysis (PRISMA) guidelines, with a predetermined search strategy [68]. Medical subject headings $(\mathrm{MeSH})$ terms were left active during the searches. There was no limit on the status, date or language of the publication. The single paper published in a language other than English was translated digitally using two separate translation software programs; Google Translate and DeepL Translator (DeepL GmbH, Cologne, Germany). The final Boolean searches were performed in PubMed and SPORTDiscus (EBSCO) on 6th May 2020. The search terms used were '(dietary supplements OR dietary supplementation OR nutritional supplements OR nutritional supplementation OR supplements OR supplementation OR ergogenic OR ergogenic aids OR caffeine OR creatine OR nitrate OR sodium bicarbonate OR beta-alanine) AND (heat OR cold OR temperature OR body temperature regulation)' and all combinations were searched independently. The dietary supplements caffeine, creatine, nitrate, sodium bicarbonate and beta-alanine were searched for individually as they have been recognised by the IOC [6] and ACSM position statements [7] as having the greatest empirical evidence for their ergogenic effects in a thermoneutral environment and are, therefore, relevant to review in the heat. As there is no a-priori list of dietary supplements that are ergogenic through their effect on thermal balance, no other supplements were searched individually by name. All relevant supplements should be identified by the other search terms. Two authors (JP and MW) verified the search terms and the accuracy of the returned results.

\subsection{Study Selection}

Following the identification of all articles, the titles and abstracts were screened for inclusion by two reviewers and any duplicates removed. 'Other sources' were also identified, such as through social media (Twitter). The reference lists of the initial papers were reviewed independently by two authors (JP and MW). The remaining articles were then assessed separately (and without influence) by JP and MW against the inclusion and exclusion criteria. There was $100 \%$ agreement in study selection between the two reviewers. Papers were required to have been published in a peerreviewed journal as original research articles with a crossover, randomised controlled trial or an independent groups design. They must also have included a control or placebo group and participants were required to be healthy adults ( $\geq 18$ years). To be included in this analysis, the studies must have passed through one of two filter points: they must have administered a dietary supplement (1) recognised by the IOC [6] and ACSM position statements [7] as having a strong evidence base for 'direct' improvements to performance; or (2) having a proposed ergogenic mechanism of action either directly or indirectly related to modifiable factors associated with thermal balance (i.e., skin blood flow, sweating, exercise efficiency). The studies must also have: (1) administered a dietary supplement (by our definition below); (2) evaluated exhaustive endurance exercise protocols performed for $\geq 75 \mathrm{~s}$; and (3) been conducted in an ambient dry-bulb temperature of $\geq 30{ }^{\circ} \mathrm{C}$ in either a laboratory or field setting. Of the remaining papers, a number were further removed for the reasons outlined in Fig. 1. These largely comprised papers that included supplements that were: co-ingested; a drug; not orally administered; or a macro-nutrient (or had a mechanism of action which was considered to be directly 


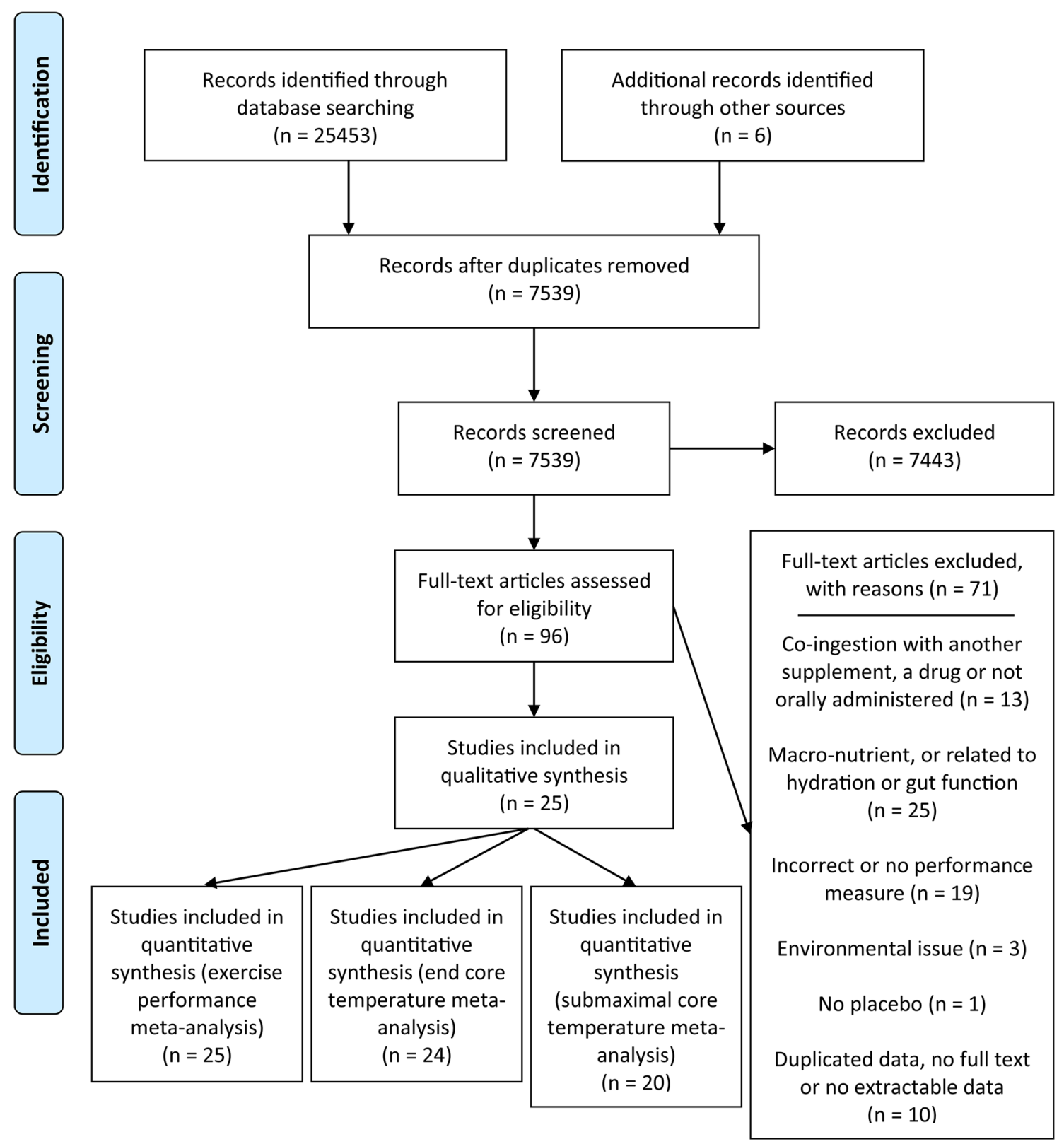

Fig. 1 Process of study selection

related to hydration or gut function). Other reasons were the absence of a performance measure or one not adhering to the above definition; or environmental issues.

We defined a dietary supplement by adapting the IOC position statement [6]: a non-food, non-pharmacological, food component, nutrient or non-food compound that is purposefully orally ingested in addition to the habitual diet with the aim to 'directly' improve sports/exercise performance. The supplement is not being consumed for its indirect health benefits, its calorific value, its effects on hydration or gut function (the ergogenic mechanism of action is not through greater fluid absorption in the gut or increased gut permeability). The supplement is also legal as per the Misuse of Drugs Act 1971 [69] and is not on the World Anti-Doping Association's prohibited substances list [70]. 'Direct' supplements refer to those acutely enhancing performance but not solely via "effective training, better recovery from training sessions, optimising mass and body composition, or reducing risks of injury and illness".

Endurance performance encompasses a variety of activities, and the current analysis allowed for three forms of exhaustive exercise of any mode; TTE, TT and power output during closed loop tasks (i.e., intermittent sprint tests [IST]). Overall effects (i.e., combinations of all modalities) were 
considered for the analysis. Any forms of exercise that were either not exhaustive or performed for $<75 \mathrm{~s}$ were removed. This was based on the knowledge that exercise performed for $\geq 75$ s has predominant contributions from aerobic metabolism, even at maximal intensities and irrespective of ambient temperature [71-73].

\subsection{Data Extraction and Quality Assessment}

Data were independently extracted on separate occasions by two authors (JP and MW) and entered into a customdesigned Microsoft Excel spreadsheet. Extracted data included: (1) characteristics of the sample (sex, age, health, training and heat acclimation/acclimatisation status); (2) study design; (3) supplement, dose and timing of intake; (4) food and fluid intake before and during exercise i.e., hydration status, food intake before exercise and fluid ingestion during exercise; (5) environmental conditions (temperature and humidity); (6) performance outcomes; (7) end and submaximal core temperature (rectal, gastrointestinal, oesophageal or tympanic); and (8) bias. Risk of bias was assessed independently by two authors (JP and MW) according to Cochrane collaboration guidelines [74]. Where details of the study were unclear, the authors of the relevant papers were consulted for specific information or to clarify the method that was used. There was 100\% agreement between the authors concerning the outcome of this quality assurance procedure; hence, it was not considered necessary to include a third independent reviewer. Standardised mean difference (SMD) was used to compare the results between studies utilising different protocols and measures. There were three outcome measures for this meta-analysis: (1) exercise performance; (2) core body temperature reported at the end of the exercise protocol, hereafter referred to as 'end core temperature'; and (3) core body temperature reported at the mid-point of the exercise protocol, hereafter referred to as 'submaximal core temperature'.

\subsection{Statistical Analysis}

Data analysis was performed by one author (JP). Data were extracted from the qualifying papers in the form of a mean, standard deviation (SD) and sample size ( $n$ ) for the metaanalysis. Publicly available software (WebPlotDigitizer, Version 4.3 [75]) was used to extrapolate any unreported values from the figures to mean and SD data. Authors of the original research articles were contacted for any missing data; however, where these were not accessible, they were imputed using the sample pooled SD from similar included studies [76]. Pre-to-post change scores were not used for any analysis, owing to their inconsistent availability. However, both sub-maximal and maximal core body temperature measures were reported to evaluate potential differences across stages of the exercise trials.

Three meta-analyses were conducted, i.e., one for each outcome measure. These were performed in RStudio [77, 78 ] and included 25, 24 and 20 comparison groups, for the exercise performance, end core temperature and submaximal core temperature meta-analyses, respectively. Not all studies reported end and submaximal core temperature; hence, they were excluded from the analysis. All data were analysed with a random-effects model, with heterogeneity assessed using the $I^{2}$ statistic. Outliers were detected using a function in RStudio and influence on analysis investigated. Publication bias was accounted for by funnel plots and conducting Egger's test and subsequently Duval and Tweedie's trim and fill procedure, when indicated [79]. Hedges' $g$ and 95\% confidence intervals (CI) were used to express SMD between dietary supplementation and placebo groups across studies. Sub-analysis of the different supplements included, and of the different exercise modalities utilised, were conducted for all three meta-analyses. Meta-regressions were also conducted to determine the effect of candidate moderators on exercise performance and core temperature outcomes, as reported in each study: training status (highly trained vs recreationally active); heat acclimation status (heat acclimated vs non-heat acclimated); hydration status (euhydrated vs hypohydrated); fluid ingestion during exercise (fluid ingestion vs no fluid ingestion); fasted vs fed state; exercise beforehand (exercise vs no exercise); heat exposure beforehand (heat exposure vs no heat exposure); duration of performance protocol; and total exercise duration. The thresholds for the magnitude of effects were $<0.2,0.2,0.5$ and 0.8 for trivial, small, medium and large effects, respectively [80]. Alpha $(\alpha)$ was set at $P \leq 0.05$ for all analyses.

\section{Results}

\subsection{Study Selection}

The initial searches retrieved 25,453 articles, plus one additional study through social media (Twitter). These were reduced to 7534 after removal of duplicates. After further screening and removal of reviews, animal studies and other irrelevant papers, 91 articles remained. Searches of the reference lists within those 91 reported studies provided five further papers. Of the 96 articles, 61 were removed based on the inclusion criteria and a further 10 were removed due to having: duplicate data with another paper, no full-text or no extractable data. This left 25 papers, of which 25,24 and 20 papers were included in the exercise performance, end core temperature and submaximal core temperature analyses, respectively (Fig. 1). 


\subsection{Study Characteristics}

The characteristics of the 25 included studies are summarised in Table 1. The studies included a total of 272 participants, comprising both males and females (males $88 \%$; both males and females 12\%) of varying training (highly trained $56 \%$; recreationally active $44 \%$ ) and heat acclimation statuses (heat acclimated 16\%; non-heated acclimated 56\%; unreported 28\%). Twenty-four of the studies had cross-over designs, while one study had an independent groups design (Table 1). Nine different types of supplements were included (caffeine, creatine, nitrate/beetroot $\left[\mathrm{NO}_{3}{ }^{-}\right]$, BCAAs, tyrosine, vitamin E, Eurycoma longifolia Jack, taurine and polyphenols) in varying doses. These were a combination of single acute doses $(n=18 ; 72 \%)$ and chronic administration $(n=7 ; 28 \%)$. The performance measures included were TT (52\%), TTE (44\%) and IST (4\%). The measures of core temperature were rectal $(64 \%)$, tympanic $(12 \%)$, oesophageal $(4 \%)$, gastrointestinal $(16 \%)$ and unreported $(4 \%)$. Ambient temperature (mean $33.2{ }^{\circ} \mathrm{C}$; range $30-42{ }^{\circ} \mathrm{C}$ ), relative humidity (mean $47 \%$; range $20-70 \%$ ) and exercise time (mean $50 \mathrm{~min}$; range 2-145 min) are reported herein. There were no adverse health-related events noted in any of the studies.

\subsection{Meta-analysis}

The results of the performance meta-analysis $(n=25)$ are reported in Fig. 2. Overall, there was a trivial significant positive effect of all supplements on exercise performance compared to placebo (Hedges' $g=0.18,95 \%$ CI $0.007-0.352$, $P=0.042)$. The $I^{2}$ statistic demonstrated $0 \%$ heterogeneity. The results of the end core temperature $(n=24)$ and submaximal core temperature $(n=20)$ meta-analyses are reported in Fig. 3. Overall, end core temperature had a small nonsignificant increase (Hedges' $g=0.20,95 \% \mathrm{CI}-0.041$ to $0.439, P=0.104)$, and submaximal core temperature had a trivial non-significant increase (Hedges' $g=0.18,95 \%$ CI -0.021 to $0.379, P=0.080$ ), with dietary supplementation compared to placebo, with $32.9 \%$ and $0 \%$ heterogeneity $\left(I^{2}\right)$, respectively.

\subsection{Sub-group Analysis}

Sub-group analyses demonstrated a non-significant effect of the different supplement categories on exercise performance $(P=0.973)$. Caffeine (Hedges' $g=0.16,95 \% \mathrm{CI}-0.123$ to $0.451, P=0.263$ ), creatine (Hedges' $g=-0.19,95 \% \mathrm{CI}$ -1.045 to $0.673, P=0.671$ ), nitrate (Hedges' $g=0.15,95 \%$ CI -0.275 to $0.574, P=0.490$ ) and polyphenols (Hedges' $g=-0.10,95 \%$ CI -0.903 to $0.698, P=0.802)$ had a trivial non-significant effect. BCAAs (Hedges' $g=0.32,95 \% \mathrm{CI}$ -0.206 to $0.851, P=0.232$ ), tyrosine (Hedges' $g=0.21$,
$95 \% \mathrm{CI}-0.288$ to $0.717, P=0.404)$, Eurycoma longifolia Jack (Hedges' $g=0.21,95 \%$ CI -0.590 to $1.016, P=0.603$ ) and vitamin E (Hedges' $g=0.22,95 \%$ CI -0.440 to 0.871 , $P=0.520$ ) had a small non-significant positive effect and taurine (Hedges' $g=0.55,95 \%$ CI -0.306 to 1.403 , $P=0.209)$ had a medium non-significant positive effect. Subgroup analysis of exercise modality (TTE, TT and IST) also demonstrated a non-significant effect of supplementation on exercise performance $(P=0.796)$. As shown in Fig. 4 , the use of any supplement had a trivial non-significant effect on TTE (Hedges' $g=0.17,95 \% \mathrm{CI}-0.077$ to $0.412, P=0.178$ ) and IST performance (Hedges' $g=-0.07,95 \% \mathrm{CI}-0.867$ to $0.734, P=0.870$ ) and a small non-significant effect on TT performance (Hedges' $g=0.22,95 \% \mathrm{CI}-0.040$ to 0.475 , $P=0.097)$.

Sub-group analysis demonstrated a significant effect of the different supplement categories on end core temperature $(P=0.003)$. Nitrate (Hedges' $g=-0.07,95 \% \mathrm{CI}-0.493$ to $0.354, P=0.748$ ), BCAAs (Hedges' $g=0.11,95 \% \mathrm{CI}$ -0.418 to $0.631, P=0.692$ ), tyrosine (Hedges' $g=0.11$, $95 \% \mathrm{CI}-0.386$ to $0.612, P=0.658)$ and Eurycoma longifolia Jack (Hedges' $g=0.00,95 \% \mathrm{CI}-0.800$ to 0.800 , $P=1.000$ ) had a trivial non-significant effect. Polyphenols (Hedges' $g=0.27,95 \% \mathrm{CI}-0.532$ to $1.078, P=0.506$ ) had a small non-significant positive effect and caffeine (Hedges' $g=0.82,95 \%$ CI $0.433-1.202, P<0.001)$ had a large significant positive effect. Vitamin E (Hedges' $g=-0.23,95 \%$ CI -0.889 to $0.423, P=0.487$ ) had a small non-significant negative effect, creatine (Hedges' $g=-0.63,95 \%$ CI -1.507 to $0.256, P=0.164$ ) had a medium non-significant negative effect and taurine (Hedges' $g=-0.96,95 \% \mathrm{CI}-1.855$ to $-0.069, P=0.035$ ) had a large significant negative effect. Sub-group analysis of exercise modality demonstrated a non-significant effect of supplementation on end core temperature $(P=0.231)$. As shown in Fig. 5, the use of any supplement had a trivial non-significant effect on TTE (Hedges' $g=-0.03,95 \% \mathrm{CI}-0.417$ to $0.355, P=0.875)$ and IST (Hedges' $g=0.16,95 \% \mathrm{CI}-0.641$ to $0.963, P=0.694$ ), but had a small significant positive effect on TT end core temperature (Hedges' $g=0.40,95 \%$ CI 0.093-0.699, $P=0.010$ ).

Sub-group analysis demonstrated a non-significant effect of the different supplement categories on submaximal core temperature $(P=0.599)$. Nitrate (Hedges' $g=-0.02,95 \% \mathrm{CI}$ -0.517 to $0.482, P=0.945$ ), BCAAs (Hedges' $g=-0.05$, 95\% CI -0.580 to $0.474, P=0.844$ ), taurine (Hedges' $g=0.06,95 \% \mathrm{CI}-0.777$ to $0.895, P=0.890)$ and vitamin $\mathrm{E}$ (Hedges' $g=0.00,95 \% \mathrm{CI}-0.653$ to $0.653, P=1.000$ ) had a trivial non-significant effect. Caffeine (Hedges' $g=0.49$, 95\% CI 0.090-0.894, $P=0.016$ ) had a small significant positive effect. Tyrosine (Hedges' $g=0.34,95 \% \mathrm{CI}-0.165$ to $0.846, P=0.187$ ) and polyphenols (Hedges' $g=0.21,95 \%$ CI -0.590 to $1.016, P=0.603$ ) had a small non-significant positive effect. Creatine (Hedges' $g=-0.27,95 \%$ CI 


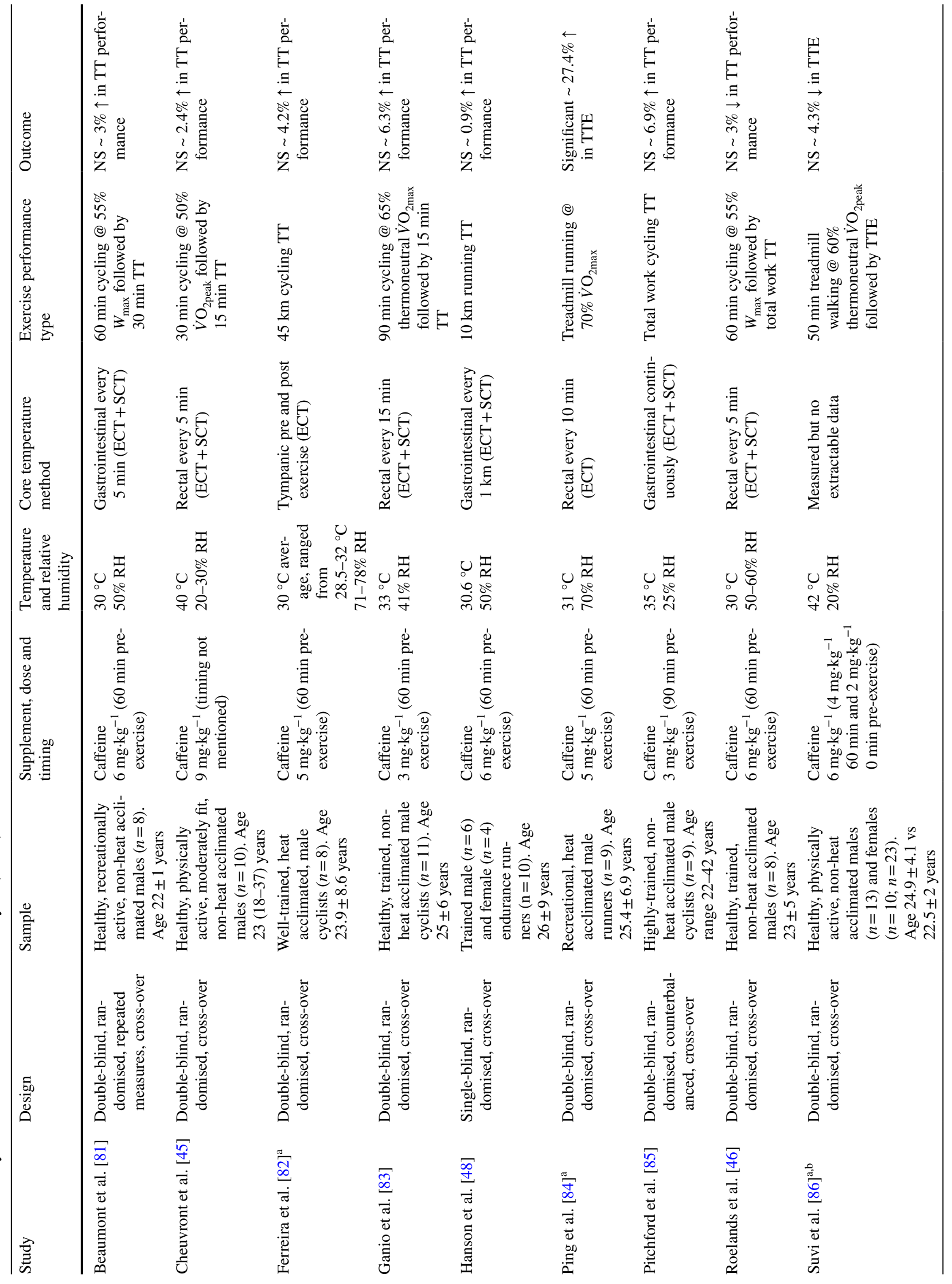




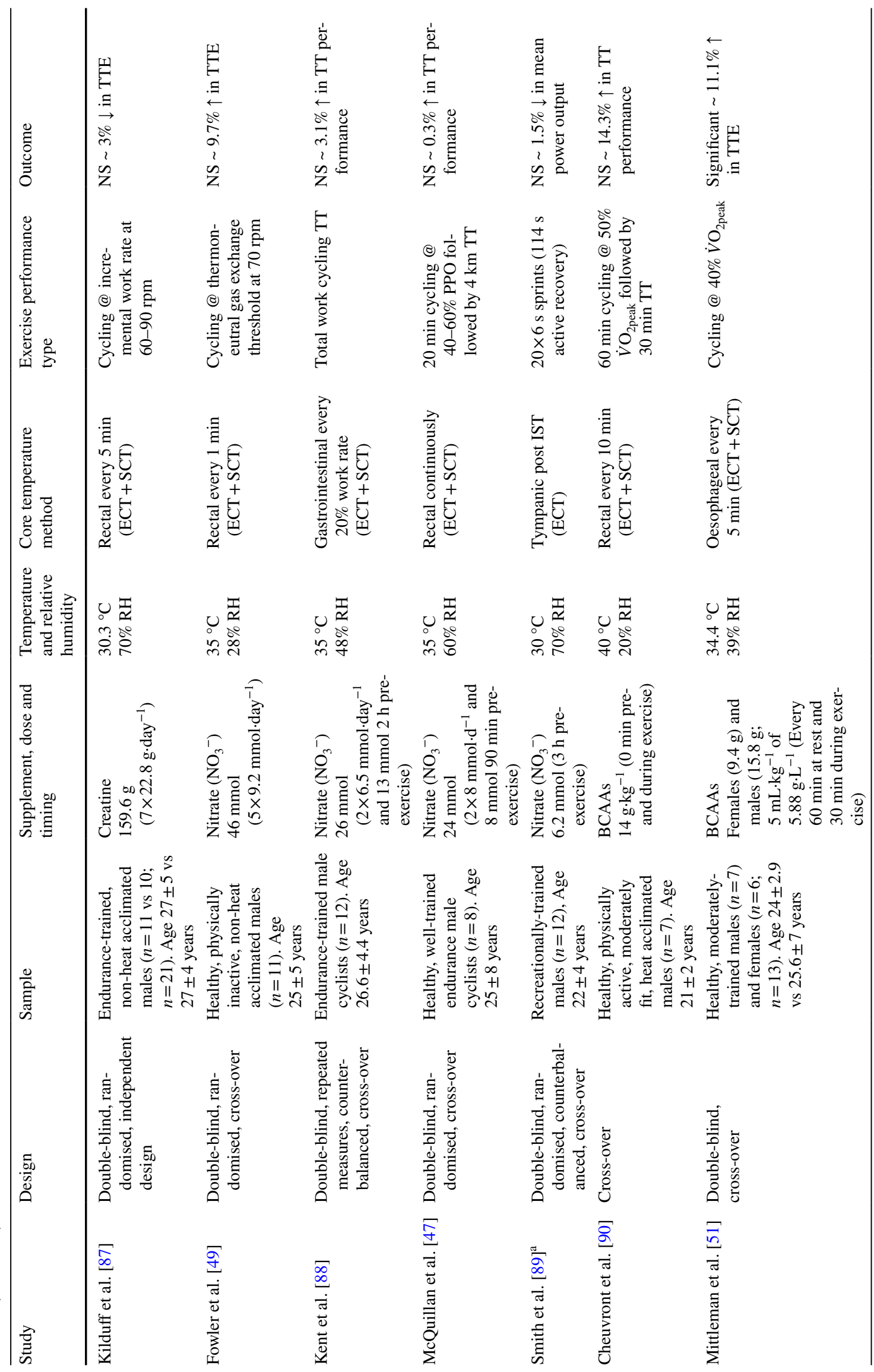




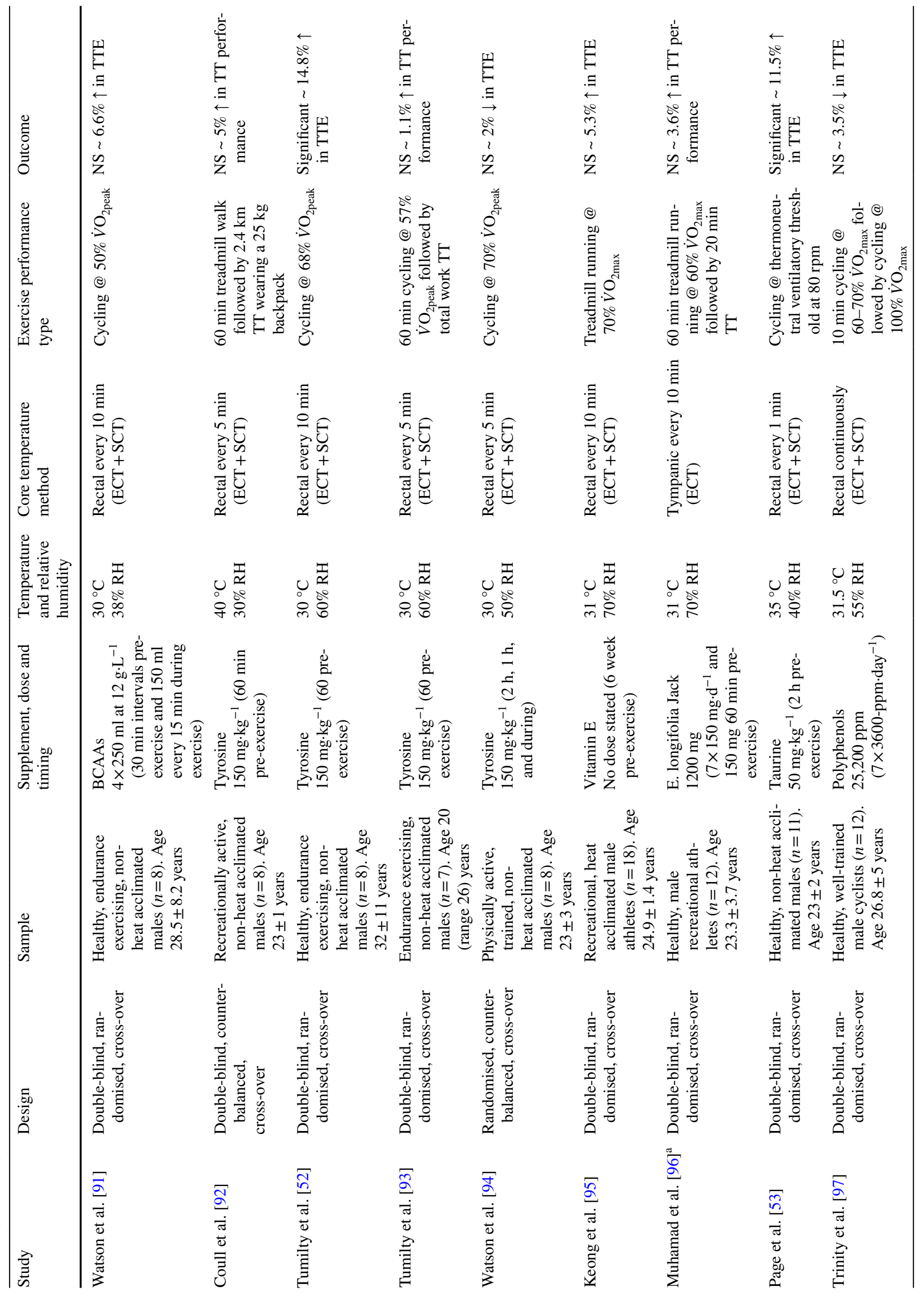


- 1.136 to $0.586, P=0.532$ ) had a small non-significant negative effect. Sub-group analysis of exercise modality also demonstrated a non-significant effect of supplementation on submaximal core temperature $(P=0.070)$. As shown in Fig. 5, the use of any supplement had a trivial non-significant effect on TTE (Hedges' $g<0.01,95 \%$ CI -0.281 to $0.278, P=0.991$ ), but had a small significant positive effect on TT submaximal core temperature (Hedges' $g=0.37,95 \%$ CI $0.082-0.654, P=0.012$ ).

\subsection{Meta-Regression}

Across the three meta-analyses, there was only one moderating effect: that of exercise before the performance protocol (exercise vs no exercise) on submaximal core temperature responses (Table 2). Otherwise, there were no significant moderating effects of any variables on the outcome of exercise performance and end core temperature or submaximal core temperature responses (Table 2).

\subsection{Risk of Bias}

The studies included had a generally 'low' or 'unclear' risk of bias, with all but three studies not stating randomisation procedures $[49,53,89]$, and two studies not adopting a blind design [90, 94]. Allocation concealment was 'unclear' in all studies (Fig. 6). There were no outliers detected and Egger's test showed that there was no publication bias in the exercise performance meta-analysis $(P=0.053)$. Several outliers [46, 53 ] were detected in the end core temperature meta-analysis, owing to the large effects certain supplements appear to have on end core temperature responses. Egger's test indicated publication bias $(P=0.015$; Fig. 7$)$, and therefore, Duval and Tweedie's trim and fill procedure was conducted, but no meaningful adjustments to the data were made. One outlier was detected in the submaximal core temperature metaanalysis [45], but no publication bias was found $(P=0.115)$.

\section{Discussion}

The main findings of the current meta-analyses were that dietary supplementation had a trivial, significant overall positive effect on endurance exercise performance in the heat (Hedges' $g=0.18, P=0.042$; Fig. 2). The secondary sub-group analysis of exercise performance revealed no differences between supplements $(P=0.973)$; however, certain supplements, such as selected AAs, demonstrated the greatest performance effect sizes in this analysis. Of particular note, caffeine (Hedges' $g=0.16, P=0.263$ ), creatine (Hedges' $g=-0.19, P=0.671$ ) and $\mathrm{NO}_{3}{ }^{-}$(Hedges' $g=0.15, P=0.490$ ) had only a trivial and non-significant effect on endurance exercise performance in the heat, despite 
Fig. 2 Effect of dietary supplementation on exercise performance

\section{Study}

SMD

$95 \%-\mathrm{Cl}$ Weight

Caffeine

Beaumont et al. [81]

Cheuvront et al. [45]

Ferreira et al. [82]

Ganio et al. [83]

Hanson et al. [48]

Ping et al. [84]

Pitchford et al. [85]

Roelands et al. [46]

Suvi et al. [86]

Random effects model $0.16[-0.12 ; 0.45]$

Heterogeneity: $I^{2}=0 \%, \tau^{2}=0, p=0.57$

\section{Creatine}

Kilduff et al. [87]

$-0.19[-1.04 ; 0.67]$

Random effects model -0.19 [-1.04; 0.67$]$

Heterogeneity: not applicable

\section{Nitrate}

Fowler et al. [49]

Kent et al. [88]

McQuillan et al. [47]

Smith et al. [89]

$0.25[-0.59 ; 1.09]$

$0.36[-0.45 ; 1.17]$

$0.02[-0.96 ; 1.00]$

$-0.07[-0.87 ; 0.73]$

Random effects model $0.15[-0.27 ; 0.57]$

Heterogeneity: $I^{2}=0 \%, \tau^{2}=0, p=0.88$

Branched-chain amino acids

Cheuvront et al. [90]

Mittleman et al. [51]

$0.39[-0.67 ; 1.45]$

$0.34[-0.44 ; 1.11]$

$0.24[-0.75 ; 1.22]$

Watson et al. [91]

$0.32[-0.21 ; 0.85]$

Heterogeneity: $I^{2}=0 \%, \tau^{2}=0, p=0.98$

\section{Tyrosine}

Coull et al. [92]

Tumilty et al. [52]

Tumilty et al. [93]

Watson et al. [94]

$0.27[-0.71 ; 1.26] \quad 3.1 \%$

$0.61[-0.40 ; 1.62] \quad 2.9 \%$

$0.05[-1.00 ; 1.10] \quad 2.7 \%$

$-0.08[-1.06 ; 0.90] \quad 3.1 \%$

Random effects model $0.21[-0.29 ; 0.72] \quad 11.8 \%$

Heterogeneity: $I^{2}=0 \%, \tau^{2}=0, p=0.79$

\section{Taurine}

Page et al. [53]

$0.55[-0.31 ; 1.40]$

Random effects model $0.55[-0.31 ; 1.40]$

Heterogeneity: not applicable

\section{Vitamin E}

Keong et al. [95]

$0.22[-0.44 ; 0.87]$

Random effects model $0.22[-0.44 ; 0.87]$

Heterogeneity: not applicable

Eurycoma longifolia Jack

Muhamad et al. [96]

$0.21[-0.59 ; 1.02]$

Random effects model 0.21 [-0.59; 1.02$]$

Heterogeneity: not applicable

Polyphenols

Trinity et al. [97]

$-0.10[-0.90 ; 0.70]$

Random effects model $-0.10[-0.90 ; 0.70]$

Heterogeneity: not applicable

Random effects model 0.18 [ $0.01 ; 0.35] 100.0 \%$

Prediction interval

$[0.00 ; 0.36]$

Heterogeneity: $I^{2}=0 \%, \tau^{2}=0, p=0.99$

Residual heterogeneity: $I^{2}=0 \%, p=0.93$
Standardised Mean Difference

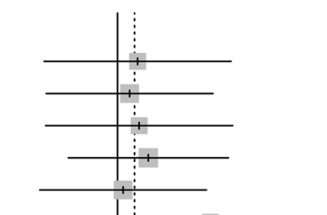

$1 \%$

$4.1 \%$

$4.2 \%$

$4.6 \%$

$3.1 \%$

$4.7 \%$

$16.6 \%$

$2.7 \%$

$5.0 \%$

$3.1 \%$

$10.7 \%$

$11.8 \%$

$4.1 \%$

$4.1 \%$

$7.0 \%$

$7.0 \%$

$4.6 \%$

$4.6 \%$

$.6 \%$
$.6 \%$

$4.7 \%$

$4.7 \%$
$\%$

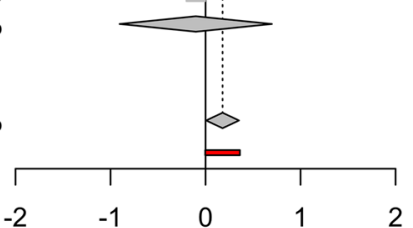

Favours placebo Favours supplement 


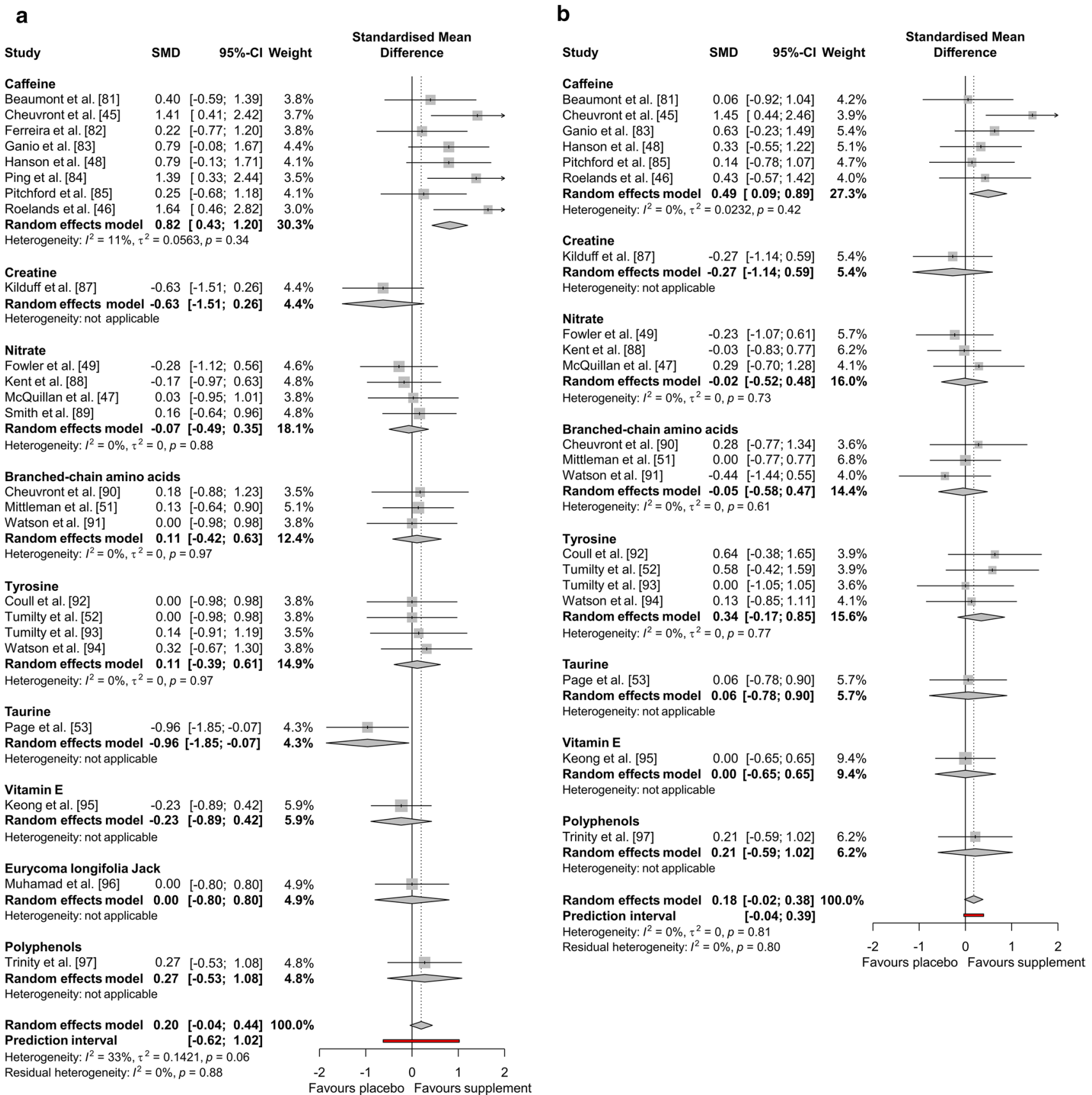

Fig. 3 Effect of dietary supplementation on $\mathbf{a}$ end core temperature and $\mathbf{b}$ submaximal core temperature

all of these supplements being recommended for athletes based on the strongest empirical evidence for performance enhancement in temperate conditions $[6,7]$. The main findings of the core temperature analyses were that, overall, dietary supplementation had a small but non-significant positive effect on end core temperature (Hedges' $g=0.20$, $P=0.104)$, and a trivial non-significant effect on submaximal core temperature (Hedges' $g=0.18, P=0.080$; Fig. 3). These results occurred irrespective of exercise duration, as demonstrated by the null effect of this moderating variable
(Table 2). The secondary sub-group analysis of end core temperature demonstrated differences between supplements $(P=0.003)$, which was largely attributable to caffeine supplementation's thermogenic effect. This evidence was surprising, given that some mechanisms underpinning the thermoneutral ergogenic effects of caffeine and $\mathrm{NO}_{3}{ }^{-}$, in particular, should, theoretically, facilitate thermal balance and performance in hot environments. These include lowered metabolic cost of exercise [98-100], peripheral vascular control $\left(\mathrm{NO}_{3}{ }^{-}[42-44]\right)$ and improved central drive (caffeine 
Study

SMD

$0.21 \quad[-0.77 ; 1.19]$

$0.13[-0.75 ; 1.00]$

$0.23[-0.76 ; 1.21]$

$0.32[-0.52 ; 1.17]$

$0.06[-0.82 ; 0.94]$

$0.75[-0.21 ; 1.72]$

$-0.24[-1.22 ; 0.75]$

$0.36[-0.45 ; 1.17]$

$0.02[-0.96 ; 1.00]$

$0.39[-0.67 ; 1.45]$

$0.27[-0.71 ; 1.26]$

$0.05[-1.00 ; 1.10]$

$0.21[-0.59 ; 1.02]$

Muhamad et al. [96]

$0.22[-0.04 ; 0.47]$

$3.1 \%$

$3.9 \%$

$3.1 \%$

$4.2 \%$

$3.9 \%$

$3.2 \%$

$3.1 \%$

$4.6 \%$

$3.1 \%$

$2.7 \%$

$3.1 \%$

$2.7 \%$

Random effects model

Heterogeneity: $I^{2}=0 \%, \tau^{2}=0, p=1.00$

\section{Time to exhaustion}

Ping et al. [84]

Suvi et al. [86]

$0.98 \quad[-0.01 ; 1.97]$

$3.0 \%$

$4.6 \%$

$45.3 \%$

\section{Standardised Mean}

Difference

Kilduff et al. [87]

Fowler et al. [49]

$-0.24[-0.82 ; 0.34]$

$8.9 \%$

$-0.19[-1.04 ; 0.67]$

$4.1 \%$

$0.25[-0.59 ; 1.09]$

$4.2 \%$

Mittleman et al. [51]

$0.34[-0.44 ; 1.11]$

$5.0 \%$

Watson et al. [91]

$0.24[-0.75 ; 1.22]$

$3.1 \%$

Tumilty et al. [52]

$0.61[-0.40 ; 1.62]$

$2.9 \%$

$-0.08[-1.06 ; 0.90]$

$3.1 \%$

Page et al. [53]

$0.55[-0.31 ; 1.40]$

$4.1 \%$

Keong et al. [95]

$0.22[-0.44 ; 0.87]$

$7.0 \%$

$-0.10[-0.90 ; 0.70]$

$4.7 \%$

Trinity et al. [97]

$0.17[-0.08 ; 0.41]$

$50.1 \%$

Heterogeneity: $I^{2}=0 \%, \tau^{2}=0, p=0.67$

Intermittent sprint test

Smith et al. [89]

$-0.07[-0.87 ; 0.73]$

$4.7 \%$

Random effects model -0.07 [-0.87; 0.73$] \quad 4.7 \%$

Heterogeneity: not applicable

$\begin{array}{lc}\text { Random effects model } & 0.18[0.01 ; 0.35] \\ \text { Prediction interval } & {[0.00 ; 0.36]}\end{array}$

Heterogeneity: $I^{2}=0 \%, \tau^{2}=0, p=0.99$

Residual heterogeneity: $I^{2}=0 \%, p=0.98$

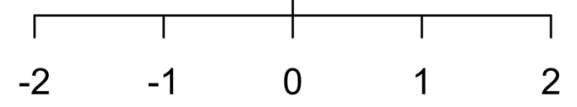

Favours placebo Favours supplement

Fig. 4 Effect of dietary supplementation on exercise performance by exercise modality

[41]). Therefore, the null findings presented herein have potentially profound implications for the use of these supplements in many performance scenarios, including major competitions or hazardous occupational settings. A possible explanation for this is that the effectiveness of otherwise established ergogenic dietary supplements is negated by the severity of hot environmental conditions. Regardless of the mechanistic reasons, these findings bring into question the 


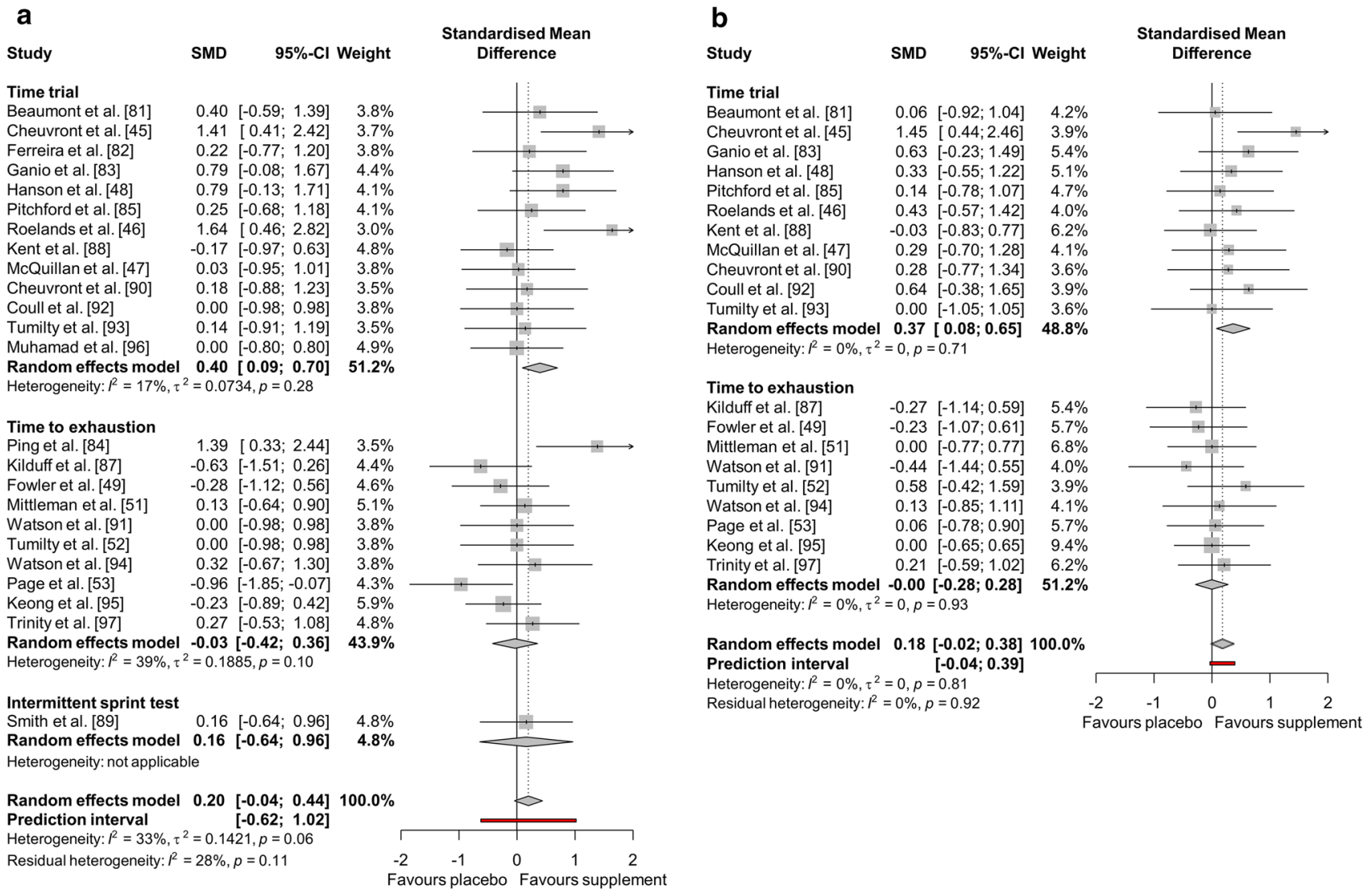

Fig. 5 Effect of dietary supplementation on $\mathbf{a}$ end core temperature and $\mathbf{b}$ submaximal core temperature by exercise modality

Table 2 Meta-regression outcomes

\begin{tabular}{llll}
\hline Moderator & Exercise performance & End core temperature response & Submaximal core temperature response \\
\hline Training status & $\beta=-0.021, P=0.907(n=25)$ & $\beta=0.095, P=0.707(n=24)$ & $\beta=-0.084, P=0.692(n=20)$ \\
Heat acclimation status & $\beta=0.247, P=0.329(n=18)$ & $\beta=0.119, P=0.770(n=17)$ & $\beta=-0.139, P=0.660(n=15)$ \\
Hydration status & $\beta=-0.153, P=0.783(n=16)$ & $\beta=-0.005, P=0.994(n=16)$ & $\beta=-0.070, P=0.909(n=12)$ \\
Fluid ingestion during exercise & $\beta=0.004, P=0.983(n=22)$ & $\beta=0.222, P=0.495(n=21)$ & $\beta=-0.082, P=0.751(n=17)$ \\
Fed vs fasted state & $\beta=0.062, P=0.763(n=19)$ & $\beta=-0.076, P=0.819(n=18)$ & $\beta=-0.064, P=0.793(n=15)$ \\
Acute heat exposure beforehand & $\beta=-0.144, P=0.416(n=25)$ & $\beta=0.384, P=0.113(n=24)$ & $\beta=0.363, P=0.082(n=20)$ \\
Exercise beforehand & $\beta=-0.183, P=0.312(n=25)$ & $\beta=0.421, P=0.089(n=24)$ & $\beta=0.449, P=0.039(n=20)$ \\
Duration of performance protocol & $\beta=0.002, P=0.532(n=24)$ & $\beta<0.001, P=0.919(n=23)$ & $\beta=-0.004, P=0.152(n=19)$ \\
Total duration of exercise & $\beta=0.002, P=0.491(n=24)$ & $\beta=0.004, P=0.247(n=23)$ & $\beta<0.001, P=0.952(n=19)$ \\
\hline
\end{tabular}

Sequence generation

Selective outcome reporting

Other threats to validity

Incomplete data outcome

Blinding

Allocation concealment

Low risk of bias

Unclear risk of bias

$\%$

$50 \%$

$50 \% \quad 75 \% \quad 100 \%$

High risk of bias

Fig. 6 Risk of bias

depth of current understanding regarding supplementation in the heat and current recommendations should be tempered by this.

The analysis of core temperature revealed that caffeine had a large (Hedges' $g=0.82, P<0.001$ ) and small (Hedges' $g=0.49, P=0.016)$ significant positive effect on end and submaximal core temperature, respectively. A significant rise in core temperature across exercise stages will deplete available heat storage capacity, leading to earlier onset of hyperthermic symptoms and reduced exercise performance 
Fig. 7 Publication bias for a exercise performance, $\mathbf{b}$ end core temperature and $\mathbf{c}$ submaximal core temperature
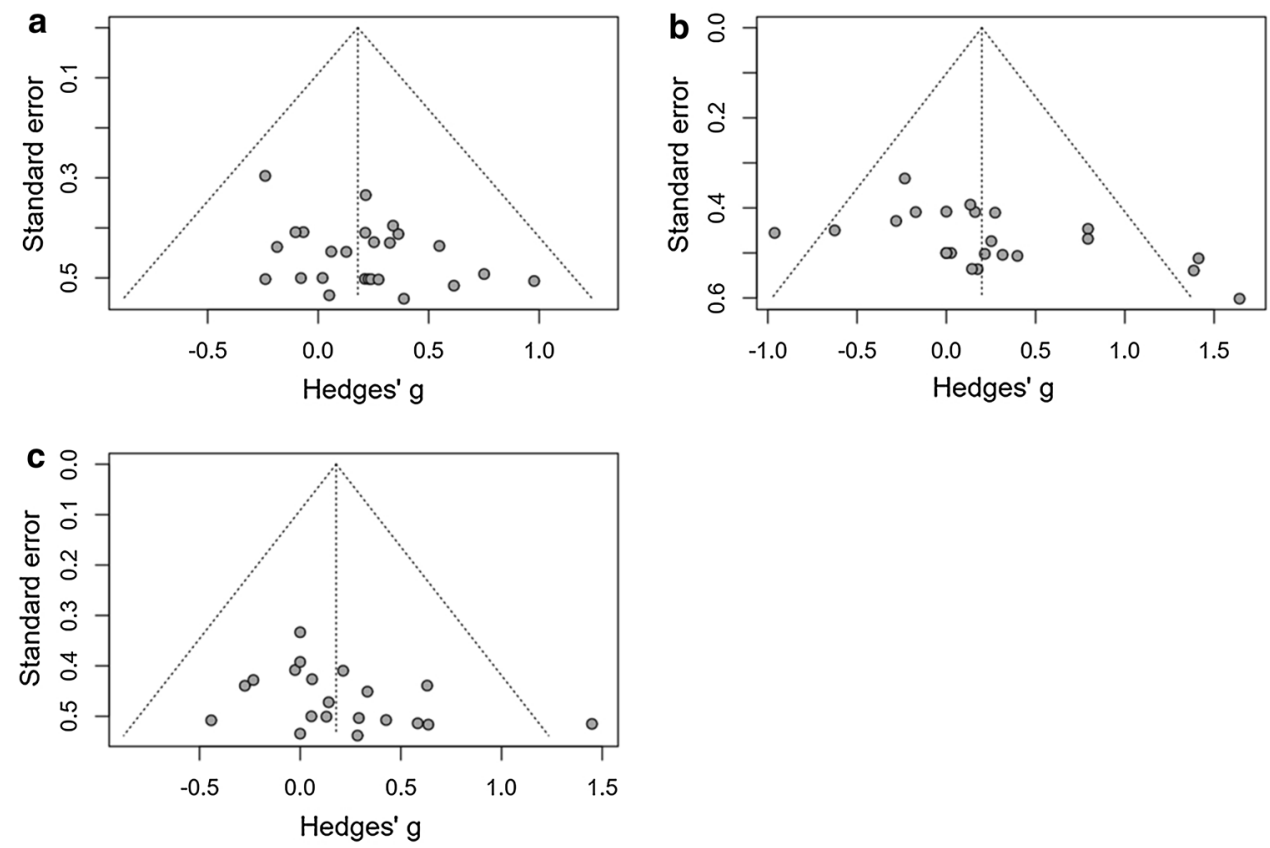

[25]. This could explain the lack of an overall ergogenic effect for caffeine. Several papers have highlighted caffeine's thermogenic effects $[45,46,48]$, but none have directly linked this to negative performance outcomes. Therefore, the current meta-analytic approach was necessary to identify this important trend across studies. Caffeine's effects are chiefly exerted via antagonism of centrally-located adenosine receptors, which act to increase the amount of circulating dopamine in the brain, as its release is inhibited by the binding of adenosine [41]. The inhibition of the reuptake of dopamine has been shown to increase core temperature [101], and therefore, a greater dopamine concentration in the brain following caffeine administration could explain the increase in core temperature demonstrated in the caffeine trials across studies. The oxygen uptake $\left(\dot{V} \mathrm{O}_{2}\right)$ response to exercise, at given exercise intensities, has also been reported to increase following caffeine ingestion compared to placebo, indicating increased metabolic heat production [102], which further supports this observation. Irrespective of any potential performance benefits, a supplement that increases core temperature when exercising in the heat could have potentially harmful effects. Given that heat illness during endurance events in hot environments is common and presents a risk to sports [34] or tactical athletes [33, 35, 36], such outcomes should be more clearly recognised in dietary guidance.

In the current meta-analysis, we also found a trivial, non-significant negative performance effect for polyphenols (Hedges' $g=-0.10, P=0.802$ ), a supplement with known anti-oxidative properties. While a small positive effect was found for the other anti-oxidants, Eurycoma longifolia Jack (Hedges' $g=0.21, P=0.603$ ) and vitamin
E (Hedges' $g=0.22, P=0.520$ ), there were no significant differences found herein or between the supplementation and placebo groups in the original research articles. Antioxidants are thought to delay fatigue by removing damaging reactive oxygen species (ROS) from the muscle and, therefore, counteracting exercise-induced oxidative stress [103]. It was somewhat unanticipated that anti-oxidants did not improve endurance exercise in the heat, since thermal stress exacerbates oxidative stress due to increased ROS production in such conditions [104]. A recent meta-analysis concluded that anti-oxidants have a moderate benefit to exercise performance in temperate conditions [105]; however, findings from individual studies remain equivocal. Studies reporting a considerable favourable effect on exercise performance administered a supra-physiological dose of $n$-acetylcysteine - a free radical scavenger - by intravenous infusion [106-108]. These findings are not supported by the majority of studies using oral anti-oxidant supplementation [109-118], with only a limited number finding a performance benefit [119-123]. It is possible that the dose and method of administration observed in the studies included in the current analysis were insufficient to elicit an ergogenic effect. In response to the current findings, further investigation into supplements conferring anti-oxidative effects in hot conditions is certainly warranted.

The supplements with the greatest ergogenic effect on exercise performance in the heat were AAs, with BCAAs (Hedges' $g=0.32, P=0.232$ ) and tyrosine (Hedges' $g=0.21$, $P=0.404)$ having a small non-significant effect, and taurine (Hedges' $g=0.55, P=0.209$ ) having a medium nonsignificant effect. While non-significant overall, the effects of AAs on exercise performance should not be discounted. 
Collectively, these supplements demonstrated the largest effect sizes, but there is currently insufficient evidence to recognise a significant effect. Interestingly, these are supplements with either equivocal or incomplete evidence for eliciting performance benefits in a thermoneutral environment [124-130]. The mechanism of action by which these AAs provide an ergogenic effect is not fully understood, but reduced central fatigue is commonly ascribed to the ergogenic effects of BCAAs and tyrosine $[52,131]$. This theory suggests that a rise in plasma free fatty acid concentration due to prolonged exercise leads to tryptophan being displaced from albumin [132]. Consequently, the plasma concentration of unbound, free tryptophan increases, resulting in greater transport across the blood-brain barrier and subsequent synthesis of serotonin [131]. This, in turn, causes lethargy, loss of drive, reduced motor unit recruitment and, ultimately, fatigue [133, 134]. Amino acids, such as BCAAs and tyrosine are thought to compete with tryptophan for transport across the blood-brain barrier, thus limiting its entry into the central nervous system, reducing the rate of serotonin synthesis and delaying fatigue $[135,136]$. Tyrosine is also a dopamine pre-cursor and dopamine plays a large role in increasing arousal, motivation and motor control [137]. Therefore, increased dopaminergic activity in the brain due to greater tyrosine concentrations may also delay fatigue, as well as increasing activation of motor pathways [138]. It is logical that these mechanisms could offset hyperthermic fatigue, as reduced central drive is observed during advanced heat stress, more so than during exercise in temperate conditions [31, 139]. However, while an overall positive effect of both BCAAs and tyrosine on performance within the current meta-analysis was demonstrated, the results of individual studies were inconsistent. The reasons for this are unclear, as while the exercise protocols, dosages (for BCAAs) and timings of ingestion differed slightly between studies, there were no apparent relationships between these variables and performance outcomes. Additional research is necessary to investigate this further.

Taurine, a sulphur containing AA, had the largest, albeit non-significant, effect on exercise performance in the heat of any of the supplements and also had a large significant negative effect on end core temperature (Hedges' $g=-0.96 P=0.035$ ). This suggests that taurine exerts a thermoregulatory effect that reduces core temperature. Page et al. [53] demonstrated that taurine increased sweating onset and rate, which might explain the improved thermal balance. These effects, in combination with taurine's capacity to enhance vasodilation [140], could facilitate both evaporative and dry heat transfer during exercise, delaying the rise in core temperature and hyperthermic fatigue. In the animal model, central infusion of taurine, a GABA agonist, has been shown to reduce core temperature in a dose-dependent manner [141]. Increased exogenous supply via oral supplementation could, therefore, offset the lower concentrations of GABA and taurine in hypothalamic nuclei following their heat stress-induced release $[142,143]$. It should be stated that only one study [53] has been conducted regarding the effect of taurine supplementation on exercise performance in the heat, and therefore, further research needs to be conducted for corroboration and further mechanistic insight.

The secondary sub-group analysis of exercise modality (TT, TTE and IST) demonstrated no effect of supplementation on endurance exercise performance, or core temperature. However, dietary supplementation did affect TT performance end core temperature and submaximal core temperature. A possible explanation for this is that the TTs included in the current analysis were generally performed at higher intensities, which is likely to elicit greater metabolic heat production and subsequent core temperature responses. Only one of the meta-regression analyses performed was significant, where pre-trial exercise moderated (increased) the submaximal core temperature outcome. This was anticipated, because prior exercise may have already raised core body temperature to some degree, thus increasing submaximal core temperature. Collectively, these results indicate that the overall thermogenic effect of dietary supplements (driven largely by caffeine) could be exacerbated by performing TTs or by performing pre-trial exercise. This could be important for athletes performing in the heat, where TT race formats are common and are often preceded by a warm-up activity $[144,145]$. Close monitoring of body temperature and other signs of heat strain might, therefore, be important if selected supplements are taken by athletes in hot TT races, alongside reduced intensity or duration of warm-up activities.

All candidate moderators, such as heat acclimation-, training-, hydration status, fluid ingestion during the trial and fed vs fasted state, did not affect exercise performance or core temperature responses to the supplements. For heat acclimation status and hydration status, this is likely due to the majority of papers mandating the recruitment of nonheat acclimated and hydrated participants. Mixed with the homogenously low effect found among most supplements in the heat, there was likely to be insufficient variation of data to establish a relationship between these variables and their effects. However, there was less consistent control of variables, such as training status, fluid ingestion during the trial, and fed vs fasted state, yet no moderating effect was found, indicating that these could not explain the variance found in any supplement's effect. On the basis of the current analysis, the effects reported could not be explained by candidate moderators but it would be useful to understand the efficacy of the most ergogenic supplements among participants of different training or acclimation statuses, given the effect of these processes on the acclimated phenotype 
[65] and the likelihood of this scenario in real-world athletic or occupational settings.

There are still a number of factors not fully investigated and which provide limitations to our current understanding of dietary supplementation for endurance exercise performance in the heat. The majority of papers used acute supplementation regimes, and therefore, the effect of chronic supplementation on exercise performance in the heat is still not well understood. Evaluation of this might be necessary for the more efficacious supplements observed here, such as taurine, and those with known benefits of chronic supplementation in thermoneutral conditions such as creatine, as this may elicit further effects. Similarly, the majority of exercise protocols were relatively short, with only nine trials exceeding $1 \mathrm{~h}$, thus limiting the current understanding of certain supplements on prolonged exercise in the heat. This is particularly important, because prolonged exercise increases the probability of heat-related illness [146], which is extremely common in some occupations, such as military settings [147]. Finally, there was a lack of 'real-world' tasks performed in the studies included in the current metaanalysis, as all but one of the studies were controlled laboratory-based investigations. Therefore, the current results need replicating in ecologically valid conditions to establish their real-world effectiveness.

\section{Conclusion}

In summary, for the first time, we have evaluated the effect of dietary supplementation on endurance exercise performance in the heat. Supplements such as caffeine and $\mathrm{NO}_{3}{ }^{-}$, which have the strongest empirical support for use in temperate conditions, lack sufficient data to support their use in the heat. Core temperature responses were also increased with caffeine supplementation, without any ergogenic benefit, which has potentially harmful health and performance consequences. Anti-oxidants also do not appear to provide a performance benefit in hot conditions. On the other hand, AAs appear to provide a greater performance benefit during exercise in the heat but the effects were often statistically insignificant. BCAAs offered the most consistent, yet small, performance effect, while taurine had both the greatest performance and thermoregulatory effect sizes of any of the supplements included in the current meta-analysis, albeit from a single study. Although further research is certainly needed, these supplements have potential to be effective for individuals exercising in hot environments. It appears that exercising in the heat significantly influences the efficacy of many dietary supplements, suggesting that findings from research conducted on certain supplements in thermoneutral conditions are not necessarily transferable to other environmental conditions. As such, research regarding the ergogenic effect of many dietary supplements for exercise in the heat is warranted. Future research should focus on understanding the mechanistic reasons for caffeine's thermogenic effects and, conversely, the thermolytic effects of taurine. The inconsistent ergogenic effects of AAs also require further investigation, as the efficacy of their use is uncertain based on the current evidence. Collectively, our findings indicate that current dietary supplementation guidelines for exercise in hot environments must be adapted and require further detail for sports and tactical personnel.

\section{Declarations}

Authorship contributions JP and MW performed the searches, screening of manuscripts and study quality scoring. JP performed the data analysis. All authors (JP, MW, MAM, SMH, RN and LPK) assisted with data interpretation and drafting of the manuscript, as well as reading and approving the final version of the manuscript.

Funding No funding was provided to support this manuscript.

Conflicts of interest/Competing interests Jennifer Peel, Melitta McNarry, Shane Heffernan, Venturino Nevola, Liam Kilduff and Mark Waldron declare that they have no conflicts of interest relevant to the content of this review.

Ethics approval Not applicable.

Consent to participate Not applicable.

Consent for publication Not applicable.

Availability of data and materials Available on request.

Code availability Not applicable.

Open Access This article is licensed under a Creative Commons Attribution 4.0 International License, which permits use, sharing, adaptation, distribution and reproduction in any medium or format, as long as you give appropriate credit to the original author(s) and the source, provide a link to the Creative Commons licence, and indicate if changes were made. The images or other third party material in this article are included in the article's Creative Commons licence, unless indicated otherwise in a credit line to the material. If material is not included in the article's Creative Commons licence and your intended use is not permitted by statutory regulation or exceeds the permitted use, you will need to obtain permission directly from the copyright holder. To view a copy of this licence, visit http://creativecommons.org/licenses/by/4.0/.

\section{References}

1. Doherty M, Smith PM. Effects of caffeine ingestion on exercise testing: a meta-analysis. Int J Sport Nutr Exerc Metab. 2004;14(6):626-46.

2. Schubert MM, Astorino TA. A systematic review of the efficacy of ergogenic aids for improving running performance. J Strength Cond Res. 2013;27(6):1699-707.

3. Christensen PM, Shirai Y, Ritz C, Nordsborg NB. Caffeine and bicarbonate for speed. A meta-analysis of legal supplements 
potential for improving intense endurance exercise performance. Front Physiol. 2017;8:240.

4. McMahon NF, Leveritt MD, Pavey TG. The effect of dietary nitrate supplementation on endurance exercise performance in healthy adults: a systematic review and meta-analysis. Sports Med. 2017;47(4):735-56.

5. Southward K, Rutherfurd-Markwick KJ, Ali A. The effect of acute caffeine ingestion on endurance performance: a systematic review and meta-analysis. Sports Med. 2018;48(8):1913-28.

6. Maughan RJ, Burke LM, Dvorak J, Larson-Meyer DE, Peeling P, Phillips SM, et al. IOC consensus statement: dietary supplements and the high-performance athlete. Int J Sport Nutr Exerc Metab. 2018;28(2):104-25.

7. Thomas DT, Erdman KA, Burke LM. Nutrition and athletic performance. Med Sci Sports Exerc. 2016;48(3):543-68.

8. Collins J, Maughan RJ, Gleeson M, Bilsborough J, Jeukendrup A, Morton JP, et al. UEFA expert group statement on nutrition in elite football. Current evidence to inform practical recommendations and guide future research. Br J Sports Med. 2020. https:// doi.org/10.1136/bjsports-2019-101961.

9. U.S. Army. FM 7-22 holistic health and fitness. Department of the Army. 2020. https://armypubs.army.mil/epubs/DR_pubs/ DR_a/ARN30714-FM_7-22-000-WEB-1.pdf. Accessed 16 Oct 2020.

10. Casey A, Hughes J, Izard RM, Greeves JP. Supplement use by UK-based British Army soldiers in training. Br J Nutr. 2014;112(7):1175-84.

11. Austin KG, McLellan TM, Farina EK, McGraw SM, Lieberman HR. Soldier use of dietary supplements, including protein and body building supplements, in a combat zone is different than use in garrison. Appl Physiol Nutr Metab. 2016;41(1):88-95.

12. Boos C, Wheble G, Campbell M, Tabner K, Woods D. Selfadministration of exercise and dietary supplements in deployed British military personnel during Operation TELIC 13. BMJ Mil Health. 2010;156(1):32-6.

13. Boos C, Simms P, Morris F, Fertout M. The use of exercise and dietary supplements among British soldiers in Afghanistan. BMJ Mil Health. 2011;157(3):229-32.

14. Ely MR, Martin DE, Cheuvront SN, Montain SJ. Effect of ambient temperature on marathon pacing is dependent on runner ability. Med Sci Sports Exerc. 2008;40(9):1675-80.

15. Racinais S, Alonso J-M, Coutts AJ, Flouris AD, Girard O, González-Alonso J, et al. Consensus recommendations on training and competing in the heat. Scand J Med Sci Sports. 2015;25:6-19.

16. Kashimura O, Minami K, Hoshi A. Prediction of WBGT for the Tokyo 2020 Olympic marathon. Japan J Biometeorol. 2016;53(4):139-44.

17. Gerrett N, Kingma BR, Sluijter R, Daanen HA. Ambient conditions prior to Tokyo 2020 Olympic and Paralympic games: considerations for acclimation or acclimatization strategies. Front Physiol. 2019;10:414.

18. World MJ, Booth TC. Iraq: the environmental challenge to HM Land Forces. Clin Med. 2008;8(4):399-403.

19. Parsons IT, Stacey MJ, Woods DR. Heat adaptation in military personnel: mitigating risk, maximizing performance. Front Physiol. 2019;10:1485.

20. Galloway S, Maughan RJ. Effects of ambient temperature on the capacity to perform prolonged cycle exercise in man. Med Sci Sports Exerc. 1997;29(9):1240-9.

21. Hargreaves M. Physiological limits to exercise performance in the heat. J Sci Med Sport. 2008;11(1):66-71.

22. Maughan RJ, Otani H, Watson P. Influence of relative humidity on prolonged exercise capacity in a warm environment. Eur $\mathbf{J}$ Appl Physiol. 2012;112(6):2313-21.
23. Junge N, Jørgensen R, Flouris AD, Nybo L. Prolonged self-paced exercise in the heat-environmental factors affecting performance. Temperature. 2016;3(4):539-48.

24. Hargreaves M, Febbraio M. Limits to exercise performance in the heat. Int J Sports Med. 1998;19:115-6.

25. González-Alonso J, Teller C, Andersen SL, Jensen FB, Hyldig $\mathrm{T}$, Nielsen B. Influence of body temperature on the development of fatigue during prolonged exercise in the heat. J Appl Physiol. 1999;86(3):1032-9.

26. Tucker R, Rauch L, Harley YX, Noakes TD. Impaired exercise performance in the heat is associated with an anticipatory reduction in skeletal muscle recruitment. Pflugers Arch. 2004;448(4):422-30.

27. Thompson MW. Cardiovascular drift and critical core temperature: factors limiting endurance performance in the heat? J Exerc Sci Fit. 2006;4(1):15-24.

28. González-Alonso J, Crandall CG, Johnson JM. The cardiovascular challenge of exercising in the heat. J Physiol. 2008;586(1):45-53.

29. Cheuvront SN, Kenefick RW, Montain SJ, Sawka MN. Mechanisms of aerobic performance impairment with heat stress and dehydration. J Appl Physiol. 2010;109(6):1989-95.

30. Nybo L. CNS fatigue provoked by prolonged exercise in the heat. Front Biosci. 2010;2:779-92.

31. Nybo L, Rasmussen P, Sawka MN. Performance in the heatphysiological factors of importance for hyperthermia-induced fatigue. Compr Physiol. 2011;4(2):657-89.

32. Périard JD, Cramer MN, Chapman PG, Caillaud C, Thompson MW. Cardiovascular strain impairs prolonged self-paced exercise in the heat. Exp Physiol. 2011;96(2):134-44.

33. Bricknell M. Heat illness in the army in Cyprus. Occup Med. 1996;46(4):304-12.

34. Howe AS, Boden BP. Heat-related illness in athletes. Am J Sports Med. 2007;35(8):1384-95.

35. Cox AT, Lentaigne J, White S, Burns D, Parsons I, O'Shea M, et al. A 2-year review of the general internal medicine admissions to the British Role 3 Hospital in Camp Bastion. Afghanistan BMJ Mil Health. 2016;162(1):56-62.

36. Armed FHSB. Update: Heat illness, active component, US Armed Forces, 2016. MSMR. 2017;24(3):9-13.

37. Benzinger T, Pratt A, Kitzinger C. The thermostatic control of human metabolic heat production. Proc Natl Acad Sci USA. 1961;47(5):730-9.

38. Gagge A, Gonzales R. Mechanisms of heat exchange: biophysics and physiology. In: Blatteis CM, Fregley MJ, editors. Handbook of physiology. Environmental physiology. Bethesda, MD: American Physiological Society; 1996. pp. 45-84.

39. Wendt D, van Loon LJ, Lichtenbelt WDM. Thermoregulation during exercise in the heat. Sports Med. 2007;37(8):669-82.

40. Ganio MS, Klau JF, Casa DJ, Armstrong LE, Maresh CM. Effect of caffeine on sport-specific endurance performance: a systematic review. J Strength Cond Res. 2009;23(1):315-24.

41. Davis JM, Zhao Z, Stock HS, Mehl KA, Buggy J, Hand GA. Central nervous system effects of caffeine and adenosine on fatigue. Am J Physiol Regul Integr Comp Physiol. 2003;284:399-404.

42. Welch G, Foote KM, Hansen C, Mack GW. Nonselective NOS inhibition blunts the sweat response to exercise in a warm environment. J Appl Physiol. 2009;106(3):796-803.

43. Fujii N, McGinn R, Stapleton JM, Paull G, Meade RD, Kenny GP. Evidence for cyclooxygenase-dependent sweating in young males during intermittent exercise in the heat. J Physiol. 2014;592(23):5327-39.

44. McGinn R, Paull G, Meade RD, Fujii N, Kenny GP. Mechanisms underlying the postexercise baroreceptor-mediated suppression of heat loss. Physiol Rep. 2014;2(10):e12168. 
45. Cheuvront SN, Ely BR, Kenefick RW, Michniak-Kohn BB, Rood JC, Sawka MN. No effect of nutritional adenosine receptor antagonists on exercise performance in the heat. Am J Physiol Regul Integr Comp Physiol. 2009;296(2):R394-401.

46. Roelands B, Buyse L, Pauwels F, Delbeke F, Deventer K, Meeusen R. No effect of caffeine on exercise performance in high ambient temperature. Eur J Appl Physiol. 2011;111(12):3089-95.

47. McQuillan JA, Casadio JR, Dulson DK, Laursen PB, Kilding AE. The effect of nitrate supplementation on cycling performance in the heat in well-trained cyclists. Int J Sports Physiol Perform. 2018;13(1):50-6.

48. Hanson NJ, Martinez SC, Byl EN, Maceri RM, Miller MG. Increased rate of heat storage, and no performance benefits, with caffeine ingestion before a $10-\mathrm{km}$ run in hot, humid conditions. Int J Sports Physiol Perform. 2019;14(2):196-202.

49. Fowler R, Jeffries O, Tallent J, Theis N, Heffernan SM, McNarry MA, et al. No thermoregulatory or ergogenic effect of dietary nitrate among physically inactive males, exercising above gas exchange threshold in hot and dry conditions. Eur J Sport Sci. 2020;21(3):370-8.

50. Amano T, Okushima D, Breese BC, Bailey SJ, Koga S, Kondo $\mathrm{N}$. Influence of dietary nitrate supplementation on local sweating and cutaneous vascular responses during exercise in a hot environment. Eur J Appl Physiol. 2018;118(8):1579-88.

51. Mittleman KD, Ricci MR, Bailey SP. Branched-chain amino acids prolong exercise during heat stress in men and women. Med Sci Sports Exerc. 1998;30(1):83-91.

52. Tumilty L, Davison G, Beckmann M, Thatcher R. Oral tyrosine supplementation improves exercise capacity in the heat. Eur $\mathbf{J}$ Appl Physiol. 2011;111(12):2941-50.

53. Page LK, Jeffries $\mathrm{O}$, Waldron M. Acute taurine supplementation enhances thermoregulation and endurance cycling performance in the heat. Eur J Sport Sci. 2019;19(8):1101-9.

54. Knapik JJ, Steelman RA, Hoedebecke SS, Austin KG, Farina EK, Lieberman HR. Prevalence of dietary supplement use by athletes: systematic review and meta-analysis. Sports Med. 2016;46(1):103-23.

55. Wardenaar FC, Ceelen IJ, Van Dijk J-W, Hangelbroek RW, Van Roy L, Van der Pouw B, et al. Nutritional supplement use by Dutch elite and sub-elite athletes: does receiving dietary counseling make a difference? Int J Sport Nutr Exerc Metab. 2017;27(1):32-42.

56. Burke LM. Nutritional needs for exercise in the heat. Comp Biochem Physiol Part A Mol Integr Physiol. 2001;128(4):735-48.

57. Carter J, Jeukendrup AE, Mundel T, Jones DA. Carbohydrate supplementation improves moderate and high-intensity exercise in the heat. Pflugers Arch. 2003;446(2):211-9.

58. Cathcart AJ, Murgatroyd SR, McNab A, Whyte LJ, Easton C. Combined carbohydrate-protein supplementation improves competitive endurance exercise performance in the heat. Eur J Appl Physiol. 2011;111(9):2051-61.

59. Sawka MN, Montain SJ, Latzka WA. Hydration effects on thermoregulation and performance in the heat. Comp Biochem Physiol Part A Mol Integr Physiol. 2001;128(4):679-90.

60. Maughan R, Shirreffs S. Exercise in the heat: challenges and opportunities. J Sports Sci. 2004;22(10):917-27.

61. Casa DJ, Stearns RL, Lopez RM, Ganio MS, McDermott BP, Walker Yeargin S, et al. Influence of hydration on physiological function and performance during trail running in the heat. J Athl Train. 2010;45(2):147-56.

62. Morris DM, Huot JR, Jetton AM, Collier SR, Utter AC. Acute sodium ingestion before exercise increases voluntary water consumption resulting in preexercise hyperhydration and improvement in exercise performance in the heat. Int J Sport Nutr Exerc Metab. 2015;25(5):456-62.
63. Tan P, Lee J. The role of fluid temperature and form on endurance performance in the heat. Scand J Med Sci Sports. 2015;25:39-51.

64. Hoffman MD, Snipe RM, Costa RJ. Ad libitum drinking adequately supports hydration during $2 \mathrm{~h}$ of running in different ambient temperatures. Eur J Appl Physiol. 2018;118(12):2687-97.

65. Ravanelli N, Coombs GB, Imbeault P, Jay O. Maximum skin wettedness after aerobic training with and without heat acclimation. Med Sci Sports Exerc. 2018;50(2):299-307.

66. Schlader ZJ, Raman A, Morton RH, Stannard SR, Mündel T. Exercise modality modulates body temperature regulation during exercise in uncompensable heat stress. Eur J Appl Physiol. 2011;111(5):757-66.

67. Racinais S, Périard JD, Karlsen A, Nybo L. Effect of heat and heat acclimatization on cycling time trial performance and pacing. Med Sci Sports Exerc. 2015;47(3):601-6.

68. Moher D, Shamseer L, Clarke M, Ghersi D, Liberati A, Petticrew $\mathrm{M}$, et al. Preferred reporting items for systematic review and meta-analysis protocols (PRISMA-P) 2015 statement. Syst Rev. $2015 ; 4(1): 1$.

69. U.K. Government. Misuse of drugs act 1971. In: U.K. public general acts. U.K. Legislation. 1971. https://www.legislation.gov. uk/ukpga/1971/38/contents. Accessed 12 May 2020.

70. World Anti-Doping Agency. Prohibited list. World Anti-Doping Agency. 2020. https://www.wada-ama.org/sites/default/files/ wada_2020_english_prohibited_list_0.pdf. Accessed 12 May 2020.

71. Gastin PB. Energy system interaction and relative contribution during maximal exercise. Sports Med. 2001;31(10):725-41.

72. Baker JS, McCormick MC, Robergs RA. Interaction among skeletal muscle metabolic energy systems during intense exercise. J Nutr Metab. 2010;2010:905612.

73. Waldron M, Villerius V, Murphy A. Augmenting performance feedback does not affect $4 \mathrm{~km}$ cycling time-trials in the heat. J Sports Sci. 2015;33(8):786-94.

74. Higgins JP, Thomas J, Chandler J, Cumpston M, Li T, Page MJ, et al. Cochrane handbook for systematic reviews of interventions. Wiley; 2019.

75. Rohatgi A. WebPlotDigitizer, Version 4.3. Pacifica, California, USA; 2017. https://automeris.io/WebPlotDigitizer. Accessed 26 July 2020.

76. Furukawa TA, Barbui C, Cipriani A, Brambilla P, Watanabe N. Imputing missing standard deviations in meta-analyses can provide accurate results. J Clin Epidemiol. 2006;59(1):7-10.

77. R Core Team. R: a language and environment for statistical computing. R foundation for statistical computing. Vienna, Austria; 2013. https://www.R-project.org/. Accessed 24 July 2020.

78. Harrer M, Cuijpers P, Furukawa T, Ebert D. Doing meta-analysis in R: a hands-on guide. PROTECT Lab Erlangen. 2019. https:// bookdown.org/MathiasHarrer/Doing_Meta_Analysis_in_R/. Accessed 28 June 2020.

79. Rothstein HR, Sutton AJ, Borenstein M. Publication bias in metaanalysis: prevention, assessment and adjustments. Sussex: Wiley; 2005.

80. Rosenthal R, Rosnow RL. Essentials of behavioral research: methods and data analysis. 3rd ed. Boston: McGraw-Hill; 2008.

81. Beaumont RE, James LJ. Effect of a moderate caffeine dose on endurance cycle performance and thermoregulation during prolonged exercise in the heat. J Sci Med Sport. 2017;20(11):1024-8.

82. Ferreira GM, Guerra GC, Guerra RO. Effect of caffeine in the performance of cyclists under high thermal risk. Acta Cir Bras. 2005;20(1):196-203.

83. Ganio MS, Johnson EC, Klau JF, Anderson JM, Casa DJ, Maresh CM, et al. Effect of ambient temperature on caffeine 
ergogenicity during endurance exercise. Eur J Appl Physiol. 2011;111(6):1135-46.

84. Ping WC, Keong CC, Bandyopadhyay A. Effects of acute supplementation of caffeine on cardiorespiratory responses during endurance running in a hot $\&$ humid climate. Indian J Med Res. 2010;132:36-41.

85. Pitchford NW, Fell JW, Leveritt MD, Desbrow B, Shing CM. Effect of caffeine on cycling time-trial performance in the heat. J Sci Med Sport. 2014;17(4):445-9.

86. Suvi S, Timpmann S, Tamm M, Aedma M, Kreegipuu K, Oopik $\mathrm{V}$. Effects of caffeine on endurance capacity and psychological state in young females and males exercising in the heat. Appl Physiol Nutr Metab. 2017;42(1):68-76.

87. Kilduff LP, Georgiades E, James N, Minnion RH, Mitchell M, Kingsmore D, et al. The effects of creatine supplementation on cardiovascular, metabolic, and thermoregulatory responses during exercise in the heat in endurance-trained humans. Int J Sport Nutr Exerc Metab. 2004;14(4):443-60.

88. Kent GL, Dawson B, Cox GR, Burke LM, Eastwood A, Croft $\mathrm{KD}$, et al. Dietary nitrate supplementation does not improve cycling time-trial performance in the heat. J Sports Sci. 2018;36(11):1204-11

89. Smith K, Muggeridge DJ, Easton C, Ross MD. An acute dose of inorganic dietary nitrate does not improve high-intensity, intermittent exercise performance in temperate or hot and humid conditions. Eur J Appl Physiol. 2019;119(3):723-33.

90. Cheuvront SN, Carter Iii R, Kolka MA, Lieberman HR, Kel$\operatorname{logg}$ MD, Sawka MN. Branched-chain amino acid supplementation and human performance when hypohydrated in the heat. J Appl Physiol. 2004;97(4):1275-82.

91. Watson P, Shirreffs SM, Maughan RJ. The effect of acute branched-chain amino acid supplementation on prolonged exercise capacity in a warm environment. Eur J Appl Physiol. 2004;93(3):306-14.

92. Coull N, Chrismas B, Watson P, Horsfall R, Taylor L. Tyrosine ingestion and its effects on cognitive and physical performance in the heat. Med Sci Sports Exerc. 2016;48(2):277-86.

93. Tumilty L, Davison G, Beckmann M, Thatcher R. Failure of oral tyrosine supplementation to improve exercise performance in the heat. Med Sci Sports Exerc. 2014;46(7):1417-25.

94. Watson P, Enever S, Page A, Stockwell J, Maughan RJ. Tyrosine supplementation does not influence the capacity to perform prolonged exercise in a warm environment. Int J Sport Nutr Exerc Metab. 2012;22(5):363-73.

95. Keong CC, Singh HJ, Singh R. Effects of palm vitamin E supplementation on exercise-induced oxidative stress and endurance performance in the heat. J Sports Sci Med. 2006;5(4):629-39.

96. Muhamad AS, Chen Chee K, Ooi Foong K, Abdullah MR, Lam CK. Effects of Eurycoma longifolia jack supplementation on recreational athletes' endurance running capacity and physiological responses in the heat. Int J Appl Sports Sci. 2010;22(2):1-19.

97. Trinity JD, Pahnke MD, Trombold JR, Coyle EF. Impact of polyphenol antioxidants on cycling performance and cardiovascular function. Nutrients. 2014;6(3):1273-92.

98. Larsen F, Weitzberg E, Lundberg J, Ekblom B. Effects of dietary nitrate on oxygen cost during exercise. Acta Physiol. 2007;191(1):59-66.

99. Larsen FJ, Weitzberg E, Lundberg JO, Ekblom B. Dietary nitrate reduces maximal oxygen consumption while maintaining work performance in maximal exercise. Free Radic Biol Med. 2010;48(2):342-7.

100. Lansley KE, Winyard PG, Fulford J, Vanhatalo A, Bailey SJ, Blackwell JR, et al. Dietary nitrate supplementation reduces the $\mathrm{O} 2$ cost of walking and running: a placebo-controlled study. J Appl Physiol. 2011;110(3):591-600.
101. Watson P, Hasegawa H, Roelands B, Piacentini MF, Looverie R, Meeusen R. Acute dopamine/noradrenaline reuptake inhibition enhances human exercise performance in warm, but not temperate conditions. J Physiol. 2005;565(3):873-83.

102. Bell DG, McLellan TM. Exercise endurance 1, 3, and $6 \mathrm{~h}$ after caffeine ingestion in caffeine users and nonusers. J Appl Physiol. 2002;93(4):1227-34.

103. Powers SK, Deruisseau KC, Quindry J, Hamilton KL. Dietary antioxidants and exercise. J Sports Sci. 2004;22(1):81-94.

104. McAnulty S, McAnulty L, Pascoe D, Gropper S, Keith R, Morrow J, et al. Hyperthermia increases exercise-induced oxidative stress. Int J Sports Med. 2005;26(3):188-92.

105. Somerville V, Bringans C, Braakhuis A. Polyphenols and performance: a systematic review and meta-analysis. Sports Med. 2017:47(8):1589-99.

106. Medved I, Brown MJ, Bjorksten AR, McKenna MJ. Effects of intravenous $\mathrm{N}$-acetylcysteine infusion on time to fatigue and potassium regulation during prolonged cycling exercise. J Appl Physiol. 2004;96(1):211-7.

107. Medved I, Brown MJ, Bjorksten AR, Murphy KT, Petersen AC, Sostaric $\mathrm{S}$, et al. $\mathrm{N}$-acetylcysteine enhances muscle cysteine and glutathione availability and attenuates fatigue during prolonged exercise in endurance-trained individuals. J Appl Physiol. 2004;97(4):1477-85.

108. McKenna MJ, Medved I, Goodman CA, Brown MJ, Bjorksten AR, Murphy KT, et al. N-acetylcysteine attenuates the decline in muscle $\mathrm{Na}+, \mathrm{K}+$-pump activity and delays fatigue during prolonged exercise in humans. J Physiol. 2006;576(1):279-88.

109. Skarpañska-Stejnborn A, Pilaczynska-Szczesniak L, Basta P, Deskur-Smielecka E, Horoszkiewicz-Hassan M. The influence of supplementation with artichoke (Cynara scolymus L.) extract on selected redox parameters in rowers. Int J Sport Nutr Exerc Metab. 2008;18(3):313-27.

110. Cureton KJ, Tomporowski PD, Singhal A, Pasley JD, Bigelman KA, Lambourne $\mathrm{K}$, et al. Dietary quercetin supplementation is not ergogenic in untrained men. J Appl Physiol. 2009;107(4):1095-104.

111. Nieman DC, Henson DA, Maxwell KR, Williams AS, McAnulty SR, Jin F, et al. Effects of quercetin and EGCG on mitochondrial biogenesis and immunity. Med Sci Sports Exerc. 2009;41(7):1467-75.

112. Utter AC, Nieman DC, Kang J, Dumke CL, Quindry JC, McAnulty SR, et al. Quercetin does not affect rating of perceived exertion in athletes during the Western States endurance run. Res Sports Med. 2009;17(2):71-83.

113. Bigelman KA, Fan EH, Chapman DP, Freese EC, Trilk JL, Cureton KJ. Effects of six weeks of quercetin supplementation on physical performance in ROTC cadets. Mil Med. 2010;175(10):791-8.

114. Kang SW, Hahn S, Kim J-K, Yang S-M, Park B-J, Lee SC. Oligomerized lychee fruit extract (OLFE) and a mixture of vitamin $\mathrm{C}$ and vitamin $\mathrm{E}$ for endurance capacity in a double blind randomized controlled trial. J Clin Biochem Nutr. 2012;50(2):106-13.

115. Askari G, Ghiasvand R, Paknahad Z, Karimian J, Rabiee K, Sharifirad G, et al. The effects of quercetin supplementation on body composition, exercise performance and muscle damage indices in athletes. Int J Prev Med. 2013;4(1):21-6.

116. Braakhuis AJ, Hopkins WG, Lowe TE. Effects of dietary antioxidants on training and performance in female runners. Eur $\mathrm{J}$ Sport Sci. 2014;14(2):160-8.

117. Scribbans TD, Ma JK, Edgett BA, Vorobej KA, Mitchell AS, Zelt JG, et al. Resveratrol supplementation does not augment performance adaptations or fibre-type-specific responses to highintensity interval training in humans. Appl Physiol Nutr Metab. 2014:39(11):1305-13. 
118. Scholten SD, Sergeev IN, Song Q, Birger CB. Effects of vitamin $\mathrm{D}$ and quercetin, alone and in combination, on cardiorespiratory fitness and muscle function in physically active male adults. Open access J Sports Med. 2015;6:229-39.

119. MacRae HS, Mefferd KM. Dietary antioxidant supplementation combined with quercetin improves cycling time trial performance. Int J Sport Nutr Exerc Metab. 2006;16(4):405-19.

120. Davis JM, Carlstedt CJ, Chen S, Carmichael MD, Murphy EA. The dietary flavonoid quercetin increases VO2max and endurance capacity. Int J Sport Nutr Exerc Metab. 2010;20(1):56-62.

121. Nieman DC, Williams AS, Shanely RA, Jin F, McAnulty SR, Triplett NT, et al. Quercetin's influence on exercise performance and muscle mitochondrial biogenesis. Med Sci Sports Exerc. 2010;42(2):338-45.

122. Roberts JD, Roberts MG, Tarpey MD, Weekes JC, Thomas CH. The effect of a decaffeinated green tea extract formula on fat oxidation, body composition and exercise performance. J Int Soc Sports Nutr. 2015;12(1):1-9.

123. Toscano LT, Tavares RL, Toscano LT, Silva CSOD, Almeida AEMD, Biasoto ACT, et al. Potential ergogenic activity of grape juice in runners. Appl Physiol Nutr Metab. 2015;40(9):899-906.

124. Van Hall G, Raaymakers J, Saris WHM, Wagenmakers AJM. Ingestion of branched-chain amino acids and tryptophan during sustained exercise in man: failure to affect performance. J Physiol. 1995;486(3):789-94.

125. Strüder H, Hollmann W, Platen P, Donike M, Gotzmann A, Weber K. Influence of paroxetine, branched-chain amino acids and tyrosine on neuroendocrine system responses and fatigue in humans. Horm Metab Res. 1998;30(4):188-94.

126. Davis J, Welsh R, De Volve K, Alderson N. Effects of branched-chain amino acids and carbohydrate on fatigue during intermittent, high-intensity running. Int J Sports Med. 1999;20(5):309-14.

127. Chinevere TD, Sawyer RD, Creer AR, Conlee RK, Parcell AC. Effects of L-tyrosine and carbohydrate ingestion on endurance exercise performance. J Appl Physiol. 2002;93(5):1590-7.

128. Sutton EE, Coll MR, Deuster PA. Ingestion of tyrosine: effects on endurance, muscle strength, and anaerobic performance. Int J Sport Nutr Exerc Metab. 2005;15(2):173-85.

129. Negro M, Giardina S, Marzani B, Marzatico F. Branched-chain amino acid supplementation does not enhance athletic performance but affects muscle recovery and the immune system. $\mathrm{J}$ Sports Med Phys Fitness. 2008;48(3):347-51.

130. Waldron M, Patterson SD, Tallent J, Jeffries O. The effects of an oral taurine dose and supplementation period on endurance exercise performance in humans: a meta-analysis. Sports Med. 2018;48(5):1247-53.

131. Newsholme EA, Blomstrand E. Branched-chain amino acids and central fatigue. J Nutr. 2006;136(1):274-6.

132. Newsholme E, Blomstrand E. Tryptophan, 5-hydroxytryptamine and a possible explanation for central fatigue. Adv Exp Med Biol. 1995;384:315-20.

133. Newsholme E, Acworth IN, Blomstrand E. Amino acids, brain neurotransmitters and a functional link between muscle and brain that is important in sustained exercise. In: Benzi G, editor. Advances in myochemistry. London: John Libbey Eurotext; 1987. p. 127-33.

134. Davis JM, Alderson NL, Welsh RS. Serotonin and central nervous system fatigue: nutritional considerations. Am J Clin Nutr. 2000;72(2):573S-S578.

135. Blomstrand E, Hassmen P, Ekblom B, Newsholme E. Administration of branched-chain amino acids during sustained exercise-effects on performance and on plasma concentration of some amino acids. Eur J Appl Physiol Occup Physiol. 1991;63(2):83-8.

136. Bongiovanni R, Kirkbride B, Newbould E, Durkalski V, Jaskiw GE. Relationships between large neutral amino acid levels in plasma, cerebrospinal fluid, brain microdialysate and brain tissue in the rat. Brain Res. 2010;1334:45-57.

137. Nestler EJ, Hyman SE, Malenka RC. Molecular neuropharmacology: a foundation for clinical neuroscience. McGraw-Hill Medical; 2001

138. Davis JM, Bailey SP. Possible mechanisms of central nervous system fatigue during exercise. Med Sci Sports Exerc. 1997;29(1):45-57.

139. Nybo L. Hyperthermia and fatigue. J Appl Physiol. 2008;104(3):871-8.

140. Sun Q, Wang B, Li Y, Sun F, Li P, Xia W, et al. Taurine supplementation lowers blood pressure and improves vascular function in prehypertension: randomized, double-blind, placebo-controlled study. Hypertension. 2016;67(3):541-9.

141. Frosini M, Sesti C, Saponara S, Ricci L, Valoti M, Palmi M, et al. A specific taurine recognition site in the rabbit brain is responsible for taurine effects on thermoregulation. Br J Pharmacol. 2003;139(3):487-94.

142. Frosini M, Sesti C, Palmi M, Valoti M, Fusi F, Mantovani P, et al. Heat-stress-induced hyperthermia alters CSF osmolality and composition in conscious rabbits. Am J Physiol Regul Integr Comp Physiol. 2000;279(6):2095-103.

143. Sharma H. Hyperthermia influences excitatory and inhibitory amino acid neurotransmitters in the central nervous system. An experimental study in the rat using behavioural, biochemical, pharmacological, and morphological approaches. J Neural Transm. 2006;113(4):497-519.

144. Hajoglou A, Foster C, De Koning JJ, Lucia A, Kernozek TW, Porcari JP. Effect of warm-up on cycle time trial performance. Med Sci Sports Exerc. 2005;37(9):1608-14.

145. Ückert S, Joch W. Effects of warm-up and precooling on endurance performance in the heat. Br J Sports Med. 2007;41(6):380-4.

146. Armstrong LE, Casa DJ, Millard-Stafford M, Moran DS, Pyne SW, Roberts WO. Exertional heat illness during training and competition. Med Sci Sports Exerc. 2007;39(3):556-72.

147. Nindl BC, Castellani JW, Warr BJ, Sharp MA, Henning PC, Spiering BA, et al. Physiological Employment Standards III: physiological challenges and consequences encountered during international military deployments. Eur J Appl Physiol. 2013;113(11):2655-72. 